\title{
A Pervasive Network Control Algorithm for Multicamera Networks
}

\author{
Sungjin Lee, Inwoong Lee, Seonghyun Kim, Sanghoon Lee, Senior Member, IEEE, and \\ Alan Conrad Bovik, Fellow, IEEE
}

\begin{abstract}
Owing to the increasingly large volume and complexity of captured videos, renewable energy systems based on solar energy are of particular interest in the design of energy harvesting (EH) wireless visual sensor networks (WVSNs). Since additional energy consumption following image capture occurs owing to image processing, mote operation, data transmission, and reception, the capture rate significantly affects the lifetime of a node. To this end, we explore a novel energy-efficient framework for EH-WVSN design by developing an optimal algorithm named capture rate and pervasive network control for multicamera networks where the quality of service is maximized by obtaining optimal values for the capture rate, allocated energy, and transmit power, based on field of view-based networking in the presence of event and power acquisition patterns. Through simulations, we demonstrate the feasibility of EH-WVSNs in terms of energy consumption, energy allocation, and capture rate in a realistic scenario (parking surveillance).
\end{abstract}

Index Terms-Capture rate, energy harvesting, visual sensor network, IEEE 802.15.4, field of view.

\section{INTRODUCTION}

A CONTINUOUS demand for reliable node intelligence has heightened interest in wireless visual sensor networks (WVSNs) that are responsive to the need for continuous and pervasive monitoring over diverse areas. Applications include urban surveillance, environmental monitoring, healthcare and battlefield visualization. WVSNs have the capability to deliver more intelligence based on autonomous node decisions and cooperative network awareness via event detection and object recognition from 2-D visual data. In order to support this expanding need for node intelligence, it is necessary to develop advanced technologies in video processing for data reduction and recognition, and in sensor node processing. This begins with video capture to transmit the bitstream, including efficient

Manuscript received September 28, 2013; revised November 26, 2013; accepted November 26, 2013. Date of publication December 11, 2013; date of current version February 21, 2014. This work was supported by the Basic Science Research Program through the National Research Foundation of Korea funded by the Ministry of Education under Grant NRF2013R1A1A2A10011764. The associate editor coordinating the review of this paper and approving it for publication was Prof. Kiseon Kim. (Corresponding author: S. Lee.)

S. Lee, I. Lee, S. Kim, and S. Lee are with the Department of Electrical and Electronic Engineering, Yonsei University, Seoul 120-749, Korea (e-mail: elflee7@yonsei.ac.kr; mayddb100@yonsei.ac.kr; sh-kim@yonsei. ac.kr; slee@yonsei.ac.kr).

A. C. Bovik is with the Laboratory for Image and Video Engineering, Department of Electrical and Computer Engineering, University of Texas at Austin, Austin, TX 78712 USA (e-mail: bovik@ece.utexas.edu).

Color versions of one or more of the figures in this paper are available online at http://ieeexplore.ieee.org.

Digital Object Identifier 10.1109/JSEN.2013.2294743 energy management, communicating with neighboring nodes for improved and integrated network intelligence, and so on.

Among these technologies, energy utilization over a limited power budget is essential to elongate the node lifetime, owing to the large volume of video data and the associated computational overhead, which is the main bottleneck of WVSN processing. Recently, as an emerging solution to alleviate this issue, renewable energy systems based on solar energy have been proposed. Energy harvesting-wireless visual sensor networks (EH-WVSNs) have the potential to enable energylimited WVSNs to operate over long periods without the need for battery replacement in outdoor environments [1]. Nevertheless, due to the large volume and inherent computational complexity of the captured videos, the design of EH-WVSNs will require new innovations to achieve the required degree of energy efficiency.

Much higher energy consumption is required to obtain an image frame from a camera as compared to typical modes of data sampling in sensor networks. Once an image frame is captured, additional energy consumption is required for the subsequent events of video processing, node operation, data transmission and reception. Based on the video capture rate, the necessary energy for the following processing events can be determined. By contrast, energy required for sensing and data sampling is largely ignored in non-video sensor networks, where the transmit and receive energies are the major parameters in the determination of the lifetime.

From image/video capture, substantial image processing can be performed, such as event detection, object recognition and image compression. The processing power and energy consumption required for this is very high. Moreover, as the data rate is increased, a higher modulation order is required, so that the energy received is significantly increased compared to the transmit energy. This received energy can no longer be regarded as a parameter to be neglected.

In regards to solar energy use in a WVSN, the power acquisition pattern from the solar cells and the event detection pattern from the captured videos are often not regular over time. Therefore, it is necessary to adjust the allocation power via an efficient energy utilization algorithm.

Finally, the captured information at each node depends on the field of view (FoV) which is a function of the position, orientation and a set of optical parameters [2]. An overlapped FoV among nodes creates visual redundancies among the images; hence further image data reduction is desirable. Information regarding FoV overlap can also be used to develop 
clustering and routing algorithms that maximize the network lifetime.

\section{A. Related Work}

There have been lots of researches on the wireless sensor networks (WSNs) and visual sensor networks (VSNs), but the researches have been performed separately. However, recently, both WSNs and VSNs have begun to be studied together [3], [4]. Several survey papers deal with the overall architecture, characteristics and research directions of WVSNs with respect to topology control, event-driven operation, image processing, communication and networking and energy management [5]-[10].

In addition, several contributions have been made to WVSN design via technical improvements ranging from analysis to implementation. In [11]-[15], low price CMOS cameras and sensor nodes (Mica-X or self-developed motes) have been developed that have low power consumption and low latency, thereby improving the feasibility of practical WVSNs. There were a few attempts to apply energy harvesting to WVSNs and sensor networks in [16]-[18], although this work was limited to optimizing the performance of mote hardware for energy efficiency.

The authors of [6] introduced a tier-based event-driven operation, wherein each node only transmits a captured image to its cluster head $(\mathrm{CH})$ node, if an event of interest is detected in the image. Although the idea is generally known in sensor network parlance as an event-based transmission, the idea can be powerfully applied to WVSNs using video analysis techniques.

Regarding energy harvesting, the authors of [19] sought to maximize the total (summed) duty cycle under the assumption of simple uniform traffic and point-to-point communication. However, it is difficult to extend these assumptions to more sophisticated WVSNs characterized by event-based traffic patterns and inter-networking amongst the sensor nodes.

To achieve energy efficient transmission, the reduction of redundant image data on WVSN was conducted in [20]-[23]. Most of this work concentrates on exploiting temporal and spatial correlations i.e., background subtraction and finding non-overlapping regions. Since it is not easy to handle the ensuing topological control and networking issues that arise from redundancy reduction, most of this work has provided only conceptual descriptions and algorithms [21]-[23].

In terms of WVSN optimization, the authors of [20] develop a method whereby, using a simple power management model, the optimal portion of the captured image is transmitted when FoVs overlap among nodes. However, they only considered the transmit energy in the optimization without including processing and receive energies. Moreover, their work relies on the existence of a strong spatial correlation among nodes, which is a considerable assumption for a WVSN.

Most other research has been limited to methods that seek to optimize the efficiency of power utilization and redundancy reduction without taking advantage of the inherent properties of the WVSN. Since the data rate of a WVSN is much higher than that of a scalar sensor network, the energy parameters must be more strongly considered in order to successfully realize practical WVSNs. The method for network optimization of WVSNs is still an open problem, yet an important one, given the plethora of significant applications awaiting WVSN deployments.

In [24], it is assumed that each camera node performs self-configuration to realize a distributed camera network where each camera node shares the FoV information with neighbor nodes, which results in a reduction of the FoV overlap among nodes. However, to achieve more intelligent networking, a hierarchical camera network topology would be more suitable for a WVSN in terms of object tracking and energy efficiency. Compared to a single layer camera network, this topology can further reduce the FoV overlap between the dense neighboring nodes and make the camera nodes operate more efficiently. For examples, the $\mathrm{CH}$ node provides a global view and the cluster slave (CS) nodes under the $\mathrm{CH}$ node provide their local views. Each CS node captures its own view whenever an event is detected. Sequentially, the $\mathrm{CH}$ node keeps monitoring the whole view after waking up. Via the flexible node operation, it is possible to optimize the camera network while recognizing the whole FoV and minimizing the energy consumption under the $\mathrm{CH}$ node owing to the multi-layer diversity. To implement such a hierarchical camera network, the network can share the camera calibration information to capture an optimal FoV among nodes through either the distributed or centralized signaling. When camera nodes are randomly deployed, it is necessary to share the FoV and camera information with each other to make the hierarchical topology in a distributed manner from the beginning. On the other hand, if they are manually deployed for a specific application, the $\mathrm{CH}$ node could be predetermined and the FoVs of CS nodes are calibrated to not overlapped before operation in the centralized manner. To increase the degree of freedom, it is assumed that the hierarchical camera network is constructed through either the distributed or centralized signaling. Once the hierarchical camera network and $\mathrm{CH}$ node are determined, the network starts operating with the $\mathrm{CH}$ node as a central node while optimizing the system parameters such as transmit power and capture rate.

In this paper, we have developed an optimal energy-efficient algorithm for EH-WVSNs named CAPTURE (Capture rAte and Pervasive neTwork control for mUlticameRa nEtworks). CAPTURE seeks to optimize the quality of service (QoS) by obtaining the optimal values for the capture rate and allocated energy and transmit power using FoV-based networking through events and power acquisition patterns from the solar cells.

\section{B. Contribution}

We arrange the main contributions as follows:

1) Power management for $E H$-WVSNs: The unique features are reflected in the analysis of an EH-WVSN over the IEEE 802.15.4 environment. In addition, the energy flow following the dictates of supply and demand is numerically described between the energy harvesting module and the power consumption module. We seek to optimize 


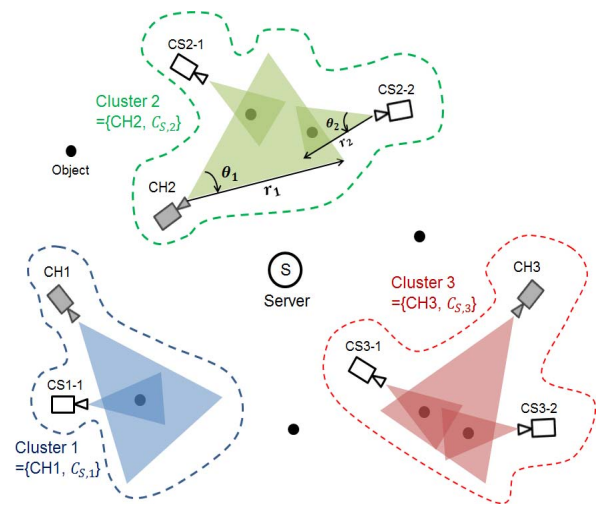

Fig. 1. Wireless camera sensor nodes based on energy harvesting using clustering.

power management to achieve realistic scenarios for EH-WVSNs.

2) Event recognition based capture rate: Contrary to conventional analyses of sensor networks, the event-driven capture rate is derived in a closed form to maximize the energy efficiency based on a tier-based hierarchical EH-WVSN topology. In particular, this event recognition based capture rate provides the high surveillance performance, with some specific patterns, in the area where the event is generated.

3) Analysis of relation between capture rate and transmit energy: Contrary to conventional analyses considering only one factor (transmit energy or capture energy), the analysis of the relation between the transmit energy and capture rate is obtained. We need to properly control the wireless and visual parameters to perform the surveillance function well.

4) FoV-based networking: The camera at each node captures a 2-D image sequence over specific viewing angles, but these images may overlap with 2-D images captured by other cameras. Node clustering is consequently performed while removing as much as possible of the internode redundancies arising from spatial correlations.

\section{SYSTEM DESCRIPTION}

\section{A. Wireless Visual Sensor Network}

Fig. 1 shows the EH-WVSN composed of three clusters. The $\mathrm{CH}$ and $\mathrm{CS}$ nodes in each cluster have their own FoV with the capture length bound $r_{h}$ and the capture angle bound $\theta_{h}$, respectively. The FoV is determined by the position, orientation, and zoom level determining the $r_{h}$ and $\theta_{h}$ of each node. $\mathcal{C}_{S, j}$ is the set of the CS nodes under the $\mathrm{CH}$ node $j$ and $\mathcal{C}_{H, k}$ is the set of $\mathrm{CH}$ nodes under the server node $k$. In this work, each cluster includes some of the CS nodes and one $\mathrm{CH}$ node and each $\mathrm{CH}$ node can track all of the events and power acquisition patterns at the $\mathrm{CS}$ nodes. Finally, each $\mathrm{CH}$ node delivers these events and power acquisition patterns to the server. Our algorithm works by using the information obtained from clustering and delivering in the hierarchical WVSNs with three levels (CS, CH, and Server).

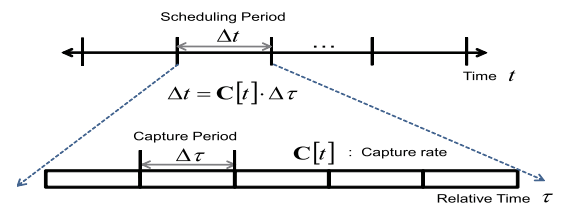

Fig. 2. Comparison of scheduling and capture periods.

\section{B. Network Clustering}

In this work, we introduce the network clustering of our system. It is assumed that each node has a camera and the nodes are independently scattered over a rectangular sensing field. It is also assumed that each node reports the calibration information (such as the position, orientation, and zoom level) to the server whenever the information is updated. Thus, the server can recognize the FoV of each node and perform the clustering even if the calibration information is changed. The flexible operation could be applied to other types of vision systems as well such as mobile camera networks [25] and reconfigurable cameras [26].

Here, the clustering relies on the overlap of FoVs among CS and $\mathrm{CH}$ nodes and on the strength of the radio signal between nodes $i$ and $j$. The clustering $\mathcal{C}_{S, j}$ for CS node $i$ is done by

$$
j^{*}=\arg \min _{j}\left\{w_{1} D_{i, j}+w_{2}\left|\mathcal{C}_{S, j}\right|\right\}
$$

subject to $\operatorname{FoV}\left(\mathbf{X}_{i}, \mathbf{O}_{i}, Z_{i}\right) \subset F o V\left(\mathbf{X}_{j}, \mathbf{O}_{j}, Z_{j}\right), \forall j \in \mathcal{C}_{H, k}$.

where $\mathbf{X}_{i}$ and $\mathbf{X}_{j}$ are the positions of CS node $i$ and $\mathrm{CH}$ node $j, \mathbf{O}_{i}$ and $\mathbf{O}_{j}$ are the orientations of $\mathrm{CS}$ node $i$ and $\mathrm{CH}$ node $j, Z_{i}$ and $Z_{j}$ are the zoom levels of CS node $i$ and $\mathrm{CH}$ node $j,\left|\mathcal{C}_{S, j}\right|$ is the cardinality of $\mathcal{C}_{S, j}$, $D_{i, j}$ is the distance between nodes $i$ and $j$, and $w_{1}$ and $w_{2}$ are the weighting factors reflecting the radio attenuation and the number of nodes. The statement $F o V\left(\mathbf{X}_{i}, \mathbf{O}_{i}, Z_{i}\right) \subset$ FoV $\left(\mathbf{X}_{j}, \mathbf{O}_{j}, Z_{j}\right)$ means that the FoV of CS node $i$ is covered by the FoV of $\mathrm{CH}$ node $j$. After clustering, each CS node belongs to one or a few clusters by reflecting the FoV overlap among the CS and $\mathrm{CH}$ nodes. Each cluster then becomes a basic cooperative unit for capturing visual information via the collaboration of nodes at the $\mathrm{CS}$ and $\mathrm{CH}$.

\section{Capture Rate}

In this work, the hierarchical timing structure for the optimal energy scheduling and for the optimal capture rate is shown in Fig. 2. The variable $t$ is the absolute time index for energy scheduling and $\Delta t$ is the time duration for each scheduling, or "scheduling period". On the other hand, $\tau$ is the time index to capture an image and $\Delta \tau$ is the time duration between two neighboring captures, or "capture period". Then, $\mathbf{C}[t]$ is defined as the capture rate at $t,\left(\mathbf{C}[t]=\frac{\Delta t}{\Delta \tau}\right)$.

\section{Node Activation}

As shown in Fig. 3, initially, each CS node captures an image, and transmits it to its $\mathrm{CH}$ node, which is used as a reference image. Thus, the CS node transmits only differences from the reference image relative to the following captured 


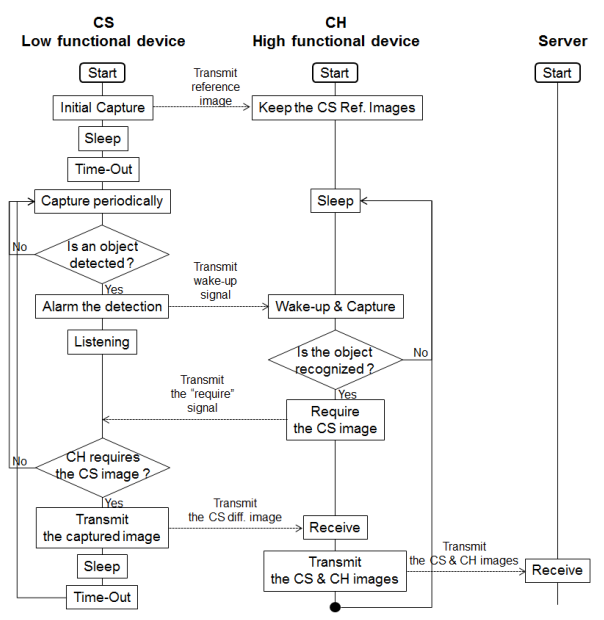

Fig. 3. Flow chart of the operation scenario.

image frames. The CS node attempts to detect objects of interest within its FoV for each captured image. If an object of interest is detected, the CS node informs the $\mathrm{CH}$ node of the fact by transmitting a notification. As soon as the $\mathrm{CH}$ node is awakened by the notification, it captures its FoV and determines whether any objects are recognized or not. If any objects are recognized at the $\mathrm{CH}$ node, it requests transmission of the difference image, and the CS node then transmits the difference image and the reference images. The $\mathrm{CH}$ node then stores one captured image taken by itself and the captured image taken by the CS node. The two images are then transmitted to the server node. If the $\mathrm{CH}$ node fails to recognize any objects from own captured image, then it does not request transmission of the difference image from the CS node.

Based on this scenario, we now define the activation processes of the $\mathrm{CS}$ and $\mathrm{CH}$ nodes. First, the activation of a CS node begins at its capture. If an object is detected in the FOV of the CS node, it informs the $\mathrm{CH}$ node of the fact and the $\mathrm{CH}$ node starts capture. Likewise, if an object is recognized as an interesting target, the $\mathrm{CH}$ node requests image transmission to the CS node. The $\mathrm{CH}$ node then transmits the two images that are captured from itself and received from the CS node. A flow chart for this procedure is shown in Fig. 3. Using the object detection probability $\left(\mathbb{P}_{d e t, i}\right)$ at node $i$ in the CS and the object recognition probability $\left(\mathbb{P}_{r e c, j}^{i}\right)$ at node $j$ in the $\mathrm{CH}$ defined by

$\mathbb{P}_{d e t, i}[t][\tau]=\{$ Probability for node $i$ to detect an object $\}$,

$\begin{aligned} \mathbb{P}_{r e c, j}^{i}[t][\tau]= & \{\text { Probability for node } j \text { to recognize } \\ & \text { the object detected at node } i\},\end{aligned}$

we define the activation probability of node $i$ in the CS as

$$
\mathbb{P}_{a c t, S}^{i}[t][\tau]=\mathbb{P}_{\text {det }, i}[t][\tau] \cdot \mathbb{P}_{r e c, j}^{i}[t][\tau] .
$$

On the other hand, the activation of the $\mathrm{CH}$ node relies on other CS nodes, since the $\mathrm{CH}$ node can be activated by any of the CS nodes under the cluster. Let $n$ be the subset of CS nodes $n \subset \mathcal{C}_{S, j}$. Each element of $n$ activates the $\mathrm{CH}$ node. Then, $n$ can be one of the possible subsets of $\mathcal{C}_{S, j}$ excluding the null set. The activation probability of the $\mathrm{CH}$ node can then be obtained using

$$
\begin{aligned}
\mathbb{P}_{a c t, H}^{j}[t][\tau]= & \sum_{n \subset \mathcal{C}_{S, j}}\left\{\prod_{i \in n} \mathbb{P}_{a c t, S}^{i}[t][\tau]\right. \\
& \left.\cdot \prod_{l \in \mathcal{C}_{S, j \backslash n}}\left(1-\mathbb{P}_{a c t, S}^{l}[t][\tau]\right)\right\},
\end{aligned}
$$

where $n \subset \mathcal{C}_{S, j}$ and $l \in \mathcal{C}_{S, j} \backslash n$ means $\left\{l: l \in \mathcal{C}_{S, j}\right.$ and $\left.l \notin n\right\}$. Similarly, the capture probability $\mathbb{P}_{c a p, j}[t][\tau]$ of the $\mathrm{CH}$ node can be derived by

$$
\begin{aligned}
\mathbb{P}_{c a p, j}[t][\tau]= & \sum_{n \subset \mathcal{C}_{S, j}}\left\{\prod_{i \in n} \mathbb{P}_{d e t, i}[t][\tau]\right. \\
& \left.\cdot \prod_{l \in \mathcal{C}_{S, j \backslash n}}\left(1-\mathbb{P}_{d e t, l}[t][\tau]\right)\right\} .
\end{aligned}
$$

\section{Networking, HARVESTING, AND REQUIRED ENERGIES}

\section{A. Allocation of Active Transmit Power}

1) Adaptation of IEEE 802.15.4 for the EH-WVSN: Based on the established documents [27], [28] regarding the wireless personal area nework (WPAN), we discuss adaptation of the superframe to the EH-WVSN.

We consider a beacon-enabled network topology under the superframe structure presented in Fig. 4, where all the abbreviations come from IEEE 802.15.4 and the related parameters. In beacon-enabled mode, each active part of a superframe is divided into 16 consecutive time slots. Moreover, the superframe duration consists of three different periods: beacon period (BP), contention access period (CAP), contention free period (CFP). In this paper, we utilize all the periods over the cluster tree topology synchronized by the personal area network (PAN) coordinator.

In particular, since communication between $\mathrm{CS}$ and $\mathrm{CH}$ nodes frequently occurs but is less important, these communications are assumed to be performed during the CAP, $t_{C A P}$, as shown in Fig. 4 . On the other hand, the communication between the $\mathrm{CH}$ and the Server is not frequent but is more important, hence the communication is assumed to be performed during the CFP as shown in $t_{C F P}$ of Fig. 4.

2) Analysis of IEEE 802.15.4: In this work, we explain the average durations of superframes and the average energy consumptions in terms of the general concept and the specific derivation. First, we conceptually define the average durations of superframes and the average energy consumptions as follows:

$$
\begin{aligned}
\bar{t}_{\text {sup }} & =\mathbb{P}_{a c t} \cdot \bar{t}_{\text {act }}+\left(1-\mathbb{P}_{\text {act }}\right) \cdot \bar{t}_{\text {inact }}, \\
\bar{E}_{\text {sup }} & =\mathbb{P}_{\text {act }} \cdot \bar{E}_{\text {act }}+\left(1-\mathbb{P}_{\text {act }}\right) \cdot \bar{E}_{\text {inact }} .
\end{aligned}
$$

The average time duration $\left(\bar{t}_{\text {sup }}\right)$ depends on the duration $\left(\bar{t}_{a c t}\right)$ when the node is active and the duration $\left(\bar{t}_{\text {inact }}\right)$ when it is inactive, weighted by the respective activation probability $\left(\mathbb{P}_{a c t}\right)$. On the other hand, the average energy consumption 


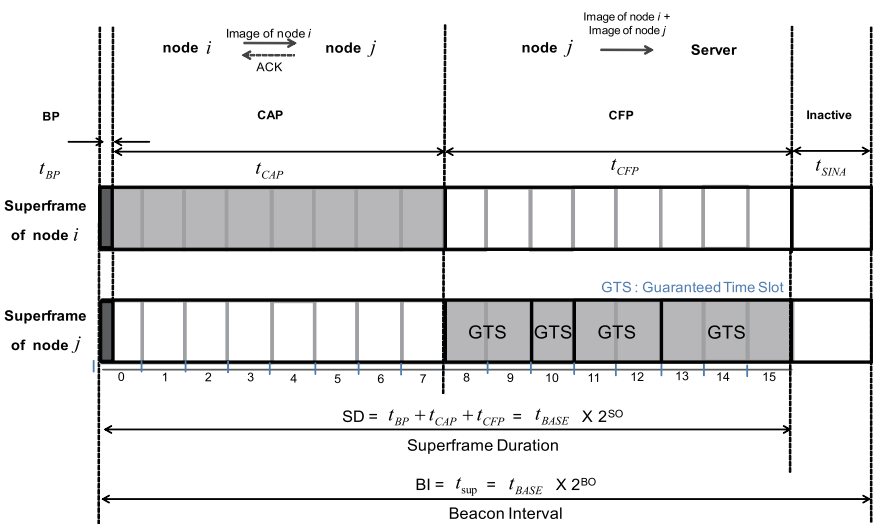

Fig. 4. Frame alignment for a hierarchical EH-WVSN using IEEE802.15.4.

$\left(\bar{E}_{\text {sup }}\right)$ depends on the consumption $\left(\bar{E}_{\text {act }}\right)$ when the node is active and the consumption $\left(\bar{E}_{\text {inact }}\right)$ when it is inactive, weighted by the respective activation probability $\left(\mathbb{P}_{a c t}\right)$.

Second, using (7) and (8), the specific average durations of superframes for nodes $i$ and $j\left(\bar{t}_{\text {sup }, S}^{i}, \bar{t}_{\text {sup }, H}^{j}\right)$ and the specific average energy consumptions $\left(\bar{E}_{\text {sup }, S}^{i}, \bar{E}_{\text {sup }, H}^{j}\right)$ are derived as shown in (9) and (10) at the bottom of the page, respectively. Here, $\bar{t}_{B P}, \bar{t}_{C A P}, \bar{t}_{C F P}$ and $\bar{t}_{S I N A}$ are the average durations of the BP, the CAP, the CFP and the inactive period, respectively, when $0 \leq S O \leq B O \leq 14$ where $S O$ and $B O$ are the abbreviation of "Superframe Order" and "Beacon Order" for representing the active duration and the superframe duration as shown in Fig. 4. Similarly, $\bar{E}_{B P}, \bar{E}_{C A P}, \bar{E}_{C F P}$ and $\bar{E}_{S I N A}$ are respectively the average energy consumptions during the $\mathrm{BP}$, the CAP, the CFP and the inactive period when $0 \leq S O \leq B O \leq 14$. On the other hand, $\bar{t}_{L I N A}$ and $\bar{E}_{L I N A}$ are the average duration and the average energy consumption over the inactive period when the object detection and object recognition events do not occur $(B O=15)$. Finally, $\mathbb{P}_{a c t, H}^{j, n}$ is the probability for the $\mathrm{CS}$ nodes to activate in the $\mathrm{CH}$ node $\mathrm{j}$, meaning the probability for the CS nodes to communicate with the $\mathrm{CH}$ node without any collision.
In (9) and (10), as shown at the bottom of the page, $t_{C A P, S}^{i}$ and $E_{C A P, S}^{i}$ indicate the duration and the energy consumption of the image transmission during the CAP from CS node $i$ to node $j$ as shown in Fig. 5(a). Due to the mechanism of CSMA-CA, when another CS node $(x \neq i)$ is activated, CS node $i$ becomes idle so that $t_{C A P, S}^{x}$ is the idle duration at the CS node during the image transmission from CS node $x$ to $\mathrm{CH}$ node $j$ and $E_{C A P, S}^{x}$ is the energy consumption during $t_{C A P, S}^{x}$.

On the other hand, $t_{C A P, H}^{n}$ and $E_{C A P, H}^{n}$ in (9) and (10) indicate the duration and the energy consumption of the image reception at the $\mathrm{CH}$ node from the CS node set $n$ as shown in Fig. 5(b). In addition, as shown in Fig. 5(c), $t_{C F P, H}^{j, n}$ and $E_{C F P, H}^{j, n}$ indicate the duration and the energy consumption at $\mathrm{CH}$ node $j$ for the image transmission from $\mathrm{CH}$ node $j$ to server node $k$. The images to be transmitted from $\mathrm{CH}$ node $j$ are composed of the images received from the CS node set $n$ and the coded image captured at $\mathrm{CH}$ node $j$. For other instances $(y \neq j), t_{C F P, H}^{y, n^{\prime}}$ is the idle duration at the $\mathrm{CH}$ node during the image transmission from $\mathrm{CH}$ node $y$ to server node $k$. Their images are composed of the images received from the CS node set $n^{\prime}$ and the coded image captured by $\mathrm{CH}$ node $y$ itself. Here, the average duration is calculated with the

$$
\begin{aligned}
\bar{t}_{\text {sup }, S}^{i}= & \mathbb{P}_{a c t, S}^{i}\left\{\bar{t}_{B P, S}^{i}+t_{C A P, S}^{i}+\sum_{x \in \mathcal{C}_{S, j} \backslash\{i\}} \mathbb{P}_{a c t, S}^{x} \cdot t_{C A P, S}^{x}+\bar{t}_{S I N A, S}^{i}\right\}+\left(1-\mathbb{P}_{a c t, S}^{i}\right) \cdot \bar{t}_{L I N A, S}^{i}, \\
\bar{t}_{\text {sup }, H}^{j}= & \sum_{n \subset \mathcal{C}_{S, j}} \mathbb{P}_{a c t, H}^{j, n}\left\{\bar{t}_{B P, H}^{j}+\sum_{y \in \mathcal{C}_{H, k} \backslash\{j\}} \sum_{n^{\prime} \subset \mathcal{C}_{S, y}} \mathbb{P}_{a c t, H}^{y, n^{\prime}} \cdot t_{C F P, H}^{y, n^{\prime}}+t_{C A P, H}^{n}+t_{C F P, H}^{j, n}+t_{S I N A, H}^{j}\right\} \\
& +\left(1-\mathbb{P}_{a c t, H}^{j}\right) \cdot \bar{t}_{L I N A, H}^{j}, \\
\bar{E}_{\text {sup }, S}^{i}= & \mathbb{P}_{a c t, S}^{i}\left\{\bar{E}_{B P, S}^{i}+E_{C A P, S}^{i}+\sum_{x \in \mathcal{C}_{S, j} \backslash\{i\}} \mathbb{P}_{a c t, S}^{x} \cdot E_{C A P, S}^{x}+\bar{E}_{S I N A, S}^{i}\right\}+\left(1-\mathbb{P}_{a c t, S}^{i}\right) \cdot \bar{E}_{L I N A, S}^{i}, \\
\bar{E}_{\text {sup }, H}^{j}= & \sum_{n \subset \mathcal{C}_{S, j}} \mathbb{P}_{a c t, H}^{j, n}\left\{\bar{E}_{B P, H}^{j}+\sum_{y \in \mathcal{C}_{H, k} \backslash\{j\}} \sum_{n^{\prime} \subset \mathcal{C}_{S, y}} \mathbb{P}_{a c t, H}^{y, n^{\prime}} \cdot E_{C F P, H}^{y, n^{\prime}}+E_{C A P, H}^{n}+E_{C F P, H}^{j, n}+E_{S I N A, H}^{j}\right\} \\
& +\left(1-\mathbb{P}_{a c t, H}^{j}\right) \cdot \bar{E}_{L I N A, H}^{j}, \\
\text { where } \quad & \mathbb{P}_{a c t, H}^{j, n}=\prod_{i \in n} \mathbb{P}_{a c t, S}^{i} \prod_{l \in \mathcal{C}_{S, j} \backslash n}\left[1-\mathbb{P}_{a c t, S}^{l}\right], \quad n \in\left\{n, n^{\prime}\right\} .
\end{aligned}
$$




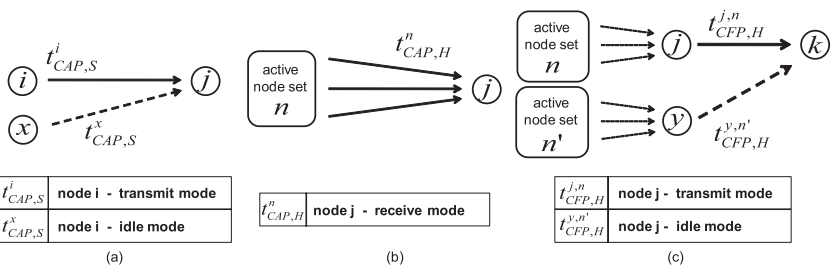

Fig. 5. Conceptual figure for the analysis of IEEE 802.15.4 superframe. (a) CAP for node i. (b) CAP for node j. (c) CFP for node $\mathrm{j}$.

activation probability $\mathrm{P}_{a c t, H}^{j, n}$ expressed as a function of the CS node set $n^{\prime}$. Likewise, $E_{C F P, H}^{y}$ is the energy consumption during $t_{C F P, H}^{y}$. The detailed analyses for the durations and energy consumptions are represented in Appendix I.

3) Power Allocation Based on IEEE 802.15.4: We can calculate the transmit powers $P_{T X, i}$ and $P_{T X, j}$ using the following average Signal to interference plus noise ratio (SINR) quality model: for CAP,

$$
\overline{\gamma_{i}}=\frac{\mathbb{P}_{a c t, S}^{i} P_{T X, i} P L(i, j)}{\sum_{y \in \mathcal{C}_{H, k}} \sum_{x \in \mathcal{C}_{S, y} \backslash\{i\}} \mathbb{P}_{a c t, S}^{x} \Gamma_{\Delta f}\left(\mu_{x}\right) P_{T X, x} P L(x, j)+N_{0}},
$$

for CFP,

$$
\overline{\gamma_{j}}=\frac{\mathbb{P}_{a c t, S}^{i} P_{T X, j} P L(j, h)}{N_{0}},
$$

where $x$ is the CS node index, $y$ is the $\mathrm{CH}$ node index, $P L(i, j)$ is the path loss over distance $d_{i, j}$ (meters) between node $i$ and node $j$, i.e. $P L(i, j)[d B]=40.2+20 \log \left(d_{i, j}\right)$, for $d_{i, j}<8$ and $P L(i, j)[d B]=58.5+33 \log \left(\frac{d_{i, j}}{8}\right)$, for $d_{i, j}$ $\geq 8$ [27]. In addition, $\mu_{x} \in$ \{channel set of IEEE 802.15.4\} is the channel selection vector of CS node $x$ and $\Gamma_{\Delta f}\left(\mu_{x}\right)$ is the frequency offset coefficient.

We can define the packet error rate (PER) as a reliability measure using the $\operatorname{SINR} \gamma_{i}$,

$$
P_{E}\left(\gamma_{i}\right)=1-\left(1-B E R\left(\gamma_{i}\right)\right)^{L}=1-\sum_{i=0}^{L}\left(\begin{array}{c}
L \\
i
\end{array}\right) B E R\left(\gamma_{i}\right)
$$

where $L$ is the length of the packet chosen in $\left\{L_{S}, L_{L}\right\}$ and $B E R\left(\gamma_{i}\right)$ is the bit error rate, $B E R\left(\gamma_{i}\right)=\frac{8}{15}$. $\frac{1}{16} \sum_{k=2}^{16}(-1)^{k}\left(\begin{array}{c}16 \\ k\end{array}\right) \exp \left(20 \cdot \gamma_{i} \cdot\left(\frac{1}{k}-1\right)\right)$ [27].

Using (10), the optimal transmit power $P_{T X}^{*}$ over the superframe can be obtained by

$$
P_{T X, i}^{*}=\arg \min _{P_{T X, i}} \bar{E}_{s u p, S \| H}^{i}\left(P_{T X, i}\right), \quad i \in\{i, j\}
$$

subject to

$$
\begin{aligned}
& P_{E}\left(\gamma_{i}\right) \leq \chi^{t h}, \\
& P_{T X, i} \in\{\text { the power modes of IEEE802.15.4\}, }
\end{aligned}
$$

where $\chi^{\text {th }}$ is the threshold PER for reliability and $P_{T X, i}$ is the transmit power at node $i$ and $\bar{E}_{\text {sup }, S \| H}^{i}$ is $\bar{E}_{\text {sup }, S}^{i}$ or $\bar{E}_{\text {sup }, H}^{j}$.

Here, $P_{T X, i}, P_{T X, j}$ should be chosen from among the limited power modes. Moreover, the optimal power levels are related to the achievable PER for a fixed data rate based on the predetermined modulation, offset quadrature phase shift keying (O-QPSK).

\section{B. Harvesting Energy}

The amount of solar intensity of light that impinges on the surface of a solar photovoltaic panel is determined by Lambert's cosine law, which states that the intensity of light $(I)$ falling on a plane is directly proportional to the cosine of the angle $(\vartheta)$ between the direction of the light source and the normal to the plane [16]: $I=k \cdot \cos \vartheta$ where $k$ is Lambert's constant. Using the above equation, we can approximately predict the harvesting energy pattern over 24 hours in a day as follows: $E_{\text {har }}[t] \cong$

$$
\begin{cases}\frac{h_{\text {peak }}}{2}\left[1+\cos \left(\frac{\pi}{6 h}(t-12 h)\right)\right] A \Delta t, & t \in[6 h, 18 h] \\ 0, & \text { otherwise }\end{cases}
$$

where $E_{\text {har }}[t]$ is the harvesting energy at time $\tau, A$ is the area of the solar panel $\left(\mathrm{cm}^{2}\right), h_{\text {peak }}$ is the peak value of the harvesting power per unit area of solar panel $\left(\mathrm{mW} / \mathrm{cm}^{2}\right)$, which may vary with season or weather, and $h$ indicates "o'clock", ex. $6 h$ means 6 o'clock; See Fig. 11(a). By modeling the harvesting pattern mathematically, more efficient scheduling can be attained.

\section{Derivation of Required Energy}

The total energy required at each node can be derived by a separate analysis of the camera operation and the networking. In particular, since the sizes of the image frames are approximately the same, the total required energy for a scheduling period can be determined based on the capture rate $\mathbf{C}[t]$ and the activation probabilities $\mathbb{P}_{a c t, S}^{i}, \mathbb{P}_{a c t, H}^{j}$, and $\mathbb{P}_{c a p, j}$. Therefore, it is possible to obtain the average required energy at node $i$ as follows,

$$
\begin{aligned}
& E_{r e q, i}[t]=\mathbf{C}[t] \cdot\left(E_{m p}+E_{d e t}\right)+\mathbf{C}[t] \cdot \bar{E}_{L I N A} \\
& +\mathbf{C}[t] \cdot \overline{\mathbb{P}}_{a c t, S}^{i}[t] \cdot\left(\bar{E}_{B P, i}+\bar{E}_{C A P, i}+\bar{E}_{S I N A, i}-\bar{E}_{L I N A}\right),
\end{aligned}
$$

where $E_{m p}$ is the energy required for mote processing (per image), $E_{d e t}$ is the energy required for object detection (per image) including processing (frame capture and frame differencing) and $\overline{\mathrm{P}}_{a c t, S}^{i}[t]$ is the average activation probability over time $t$.

Similarly, the average energy required at an intermediate node $j$ is obtained by

$$
\begin{aligned}
E_{\text {req }, j}[t]= & \mathbf{C}[t] \cdot \overline{\mathbb{P}}_{\text {cap }, j}[t] \cdot\left(E_{\text {wake }}+E_{\text {stab }}+E_{\text {init }}+E_{\text {grab }}\right. \\
& \left.+E_{\text {rec }}+E_{\text {shut }}\right)+\mathbf{C}[t] \cdot \bar{E}_{L I N A}+\mathbf{C}[t] \cdot \overline{\mathbb{P}}_{a c t, H}^{j}[t] \\
& \cdot\left(\bar{E}_{B P, j}+\bar{E}_{C A P, j}+\bar{E}_{C F P, j}+\bar{E}_{S I N A, j}-\bar{E}_{L I N A}\right),
\end{aligned}
$$

where $E_{\text {wake }}, E_{\text {stab }}, E_{\text {init }}, E_{\text {grab }}, E_{\text {rec }}$ and $E_{\text {shut }}$ are the energy per image required for wakeup, wakeup stabilization, camera initialization, frame grab, object recognition and shutdown, respectively. $\overline{\mathbb{P}}_{c a p, j}[t]$ and $\overline{\mathrm{P}}_{a c t, H}^{j}[t]$ are the average capture probability and the average activation probability over $t$. 
TABLE I

PARAMETERS USED FOR THE OPTIMIZATION OF EH-WVSN

\begin{tabular}{||c|c||c|c||}
\hline Parameter & Meaning & Parameter & Meaning \\
\hline$w_{1}$ & Weight reflecting the radio attenuation & $w_{2}$ & Weight reflecting the number of CS nodes \\
\hline $\mathcal{C}_{S, j}$ & Set of CS nodes under the CH node $j$ & $\mathcal{C}_{H, k}$ & Set of CH nodes under the server node $k$ \\
\hline $\mathrm{P}_{a c t, S}^{i}$ & Activation probability of node $i$ in CS nodes & $\mathbb{P}_{a c t, H}^{j}$ & Activation probability of node $j$ in CH nodes \\
\hline $\mathrm{P}_{c a p, j}$ & Capture probability of node $j$ in CH nodes & $P_{T X, i}$ & Transmit energy at node $i$ (Sec. III-A) \\
\hline$E_{h a r, i}[t]$ & Harvesting energy at node $i$ (Sec. III-B) & $E_{r e q, i}[t]$ & Average required energy at node $i$ (Sec. III-C) \\
\hline$E_{a l c, i}^{*}[t]$ & Allocated energy at node $i$ (Sec. IV) & $\mathbf{C}_{j}^{*}[t]$ & Capture rate at cluster of $j \mathrm{CH}$ node (Sec. V) \\
\hline
\end{tabular}

\section{Optimal Energy Scheduling}

Now we derive the optimal energy allocation $\left(E_{a l c, i}^{*}[t]\right.$, $\left.E_{a l c, j}^{*}[t]\right)$ to maximize the QoS at nodes $i$ and $j$ based on the aforementioned system analysis.

First of all, to optimally determine $E_{a l c, i}^{*}[t]$ and $E_{a l c, j}^{*}[t]$ as a function of the required energy $E_{r e q, i}[t]$ and $E_{r e q, j}[t]$ and the harvesting energy $E_{h a r, i}[t]$ and $E_{h a r, j}[t]$, we derive a utility function for $E_{a l c, i}$ and $E_{a l c, j}$ based on the proportional fairness:

$$
U_{i}[t]=\log \left(1+\overline{\mathbb{P}}_{a c t, S \| H}^{i}[t] \cdot E_{a l c, i}[t]\right), \quad i \in(i, j)
$$

where $\overline{\mathbb{P}}_{a c t, S|| H}^{i}[t]$ is $\overline{\mathbb{P}}_{a c t, S}^{i}[t]$ or $\overline{\mathbb{P}}_{a c t, H}^{j}[t]$ and $\overline{\mathbb{P}}_{a c t, S|| H}^{i}[t]$ is assigned as weight. In addition, (18) can be represented using a simpler matrix form as $\sum_{t \in\left[t_{0}, t_{0}+n_{s c} \cdot \Delta t\right]} U_{i}[t]=\mathbf{1}^{T} \mathbf{U}_{i}$ where $n_{s c}$ is the number of scheduling periods in a day, $\mathbf{1}$ is the unit column vector of size of $n_{s c}+1$ and $\mathbf{U}_{i}$ is the column vector of $U_{i}[t]$ of size $n_{s c}+1$, i.e. $\mathbf{U}_{i}=\left\{U_{i}\left[t_{0}\right], \ldots, U_{i}\left[t_{0}+n_{s c} \cdot \Delta t\right]\right\}^{T}$.

It is assumed that the energy source is limited to solar power (i.e. a Helimote), that the energy harvesting system can obtain the energy from the solar panel, and that this energy can be consumed with full efficiency or recharged with efficiency $\eta$. In addition, when the energy is directly consumed from the solar panel, no leakage occurs, i.e., $\eta=1$. On the other hand, if the energy is recharged into the battery, the efficiency falls in the range $0<\eta<1$. Thus, higher energy utilization can be achieved by direct energy consumption.

For simplicity, day-time $T_{D}$, night-time $T_{N}$ and any-time $T$ are defined by $T_{D}=\left\{\tau \mid E_{\text {har }, i}[t]>0\right\}, T_{N}=\left\{\tau \mid E_{\text {har }, i}\right.$ $[t]=0\}$ and $T=T_{D} \cup T_{N}$ at node $i$. During day-time, both recharging and expenditure of the node energy can occur simultaneously. The residual energy at time $t_{0}+n_{s c} \cdot \Delta t$ including both daytime and nighttime for that node $i$ can be modeled as

$$
\begin{aligned}
& E_{\text {res }, i}\left[t_{0}+n_{s c} \cdot \Delta t\right]=E_{\text {res }, i}\left[t_{0}\right] \\
& +\eta \sum_{t=\left[t_{0}, t_{0}+n_{s c} \cdot \Delta t\right] \subset T_{D}}\left[E_{\text {har }, i}[t]-E_{\text {alc }, i}[t]\right]^{+} \\
& -\sum_{t=\left[t_{0}, t_{0}+n_{s c} \cdot \Delta t\right] \subset T_{D}}\left[E_{\text {alc }, i}[t]-E_{\text {har }, i}[t]\right]^{+} \\
& -\sum_{t=\left[t_{0}, t_{0}+n_{s c} \cdot \Delta t\right] \subset T_{N}} E_{\text {alc }, i}[t]-\sum_{t=\left[t_{0}, t_{0}+n_{s c} \cdot \Delta t\right] \subset T} E_{\text {leak }}[t],
\end{aligned}
$$

where $E_{\text {res }}\left[t_{0}\right]$ is the residual energy at initial time $t_{0}$ and $n_{s c} \cdot \Delta t$ is the arbitrary time duration for observation. In (19), the first term is an initial energy value at the battery, the second term is the energy recharged with efficiency $\eta$ after excluding $E_{a l c, i}[t]$ for the daytime, the third term is the energy consumption of $E_{a l c, i}$ that is greater than the instantaneous harvesting energy, the fourth term is the energy allocated at $T_{N}$, and the fifth term is the leakage energy during $\left[t_{0}, t_{0}+\right.$ $\left.n_{s c} \cdot \Delta t\right]$.

For the case of the night-time only, since the harvesting energy becomes almost 0 , its residual energy can be modeled as $E_{r e s, i}\left[t_{0}+n_{s c} \cdot \Delta t\right]=E_{r e s, i}\left[t_{0}\right]-\sum_{t \subset T_{N}} E_{a l c, i}[t]-$ $\sum_{t \subset T} E_{\text {leak }}[t]$. However, since the residual energy is limited by the battery capacity $B_{\max }$, it should hold $E_{r e s, i}\left[t_{0}+n_{s c}\right.$. $\Delta t]=\min \left\{B_{\text {max }}, E_{\text {res }, i}\left[t_{0}+n_{s c} \cdot \Delta t\right]\right\}$, for $\forall n_{s c}$.

Finally, (20) is derived from (19) by assuming $E_{a l c, i}[t]<$ $E_{\text {har }, i}[t]$ for $t \in T_{D}$,

$$
\begin{aligned}
& \eta \sum_{t=\left[t_{0}, t_{0}+n_{s c} \cdot \Delta t\right] \subset T_{D}}\left[E_{\text {har }, i}[t]-E_{a l c, i}[t]\right] \\
& -\sum_{t=\left[t_{0}, t_{0}+n_{s c} \cdot \Delta t\right] \subset T_{N}} E_{\text {alc, } i}[t]-\sum_{t=\left[t_{0}, t_{0}+n_{s c} \cdot \Delta t\right] \subset T} E_{\text {leak }}[t] \\
& =\eta \mathbf{1}^{T} \mathbf{E}_{\text {har }, i}-\eta \mathbf{1}^{T} \mathbf{E}_{\text {alc }, i, T_{D}}-\mathbf{1}^{T} \mathbf{E}_{\text {alc }, i, T_{N}}-\mathbf{1}^{T} \mathbf{E}_{\text {leak }, i}=\Phi
\end{aligned}
$$

where $\Phi$ is the energy difference over the observation interval $\left[t_{0}, t_{0}+n_{s c} \cdot \Delta t\right]$. Note that with the assumption $E_{a l c, i}[t]<$ $E_{\text {har }, i}[t]$ for $t \in T_{D} \Phi$ should be non-zero to play the role of guard band to overcome estimation errors, and so on. $\mathbf{E}_{h a r, i}, \mathbf{E}_{a l c, i}$ and $\mathbf{E}_{l e a k, i}$ are the matrix expressions of $E_{\text {har }, i}[t], E_{a l c, i}[t]$ and $E_{\text {leak }, i}[t]$. In particular, $\mathbf{E}_{a l c, i, T_{D}}$ and $\mathbf{E}_{a l c, i, T_{N}}$ are the matrix expressions of $E_{a l c, i}[t]$ over $t \in T_{D}$ and $t \in T_{N}$.

By using the energy balance equation derived in (20), the optimal energy allocation problem can then be performed by controlling the variable cost $\mathbf{E}_{a c t, i}$ in order to achieve the maximum profit $\mathbf{U}_{i}$ under

$$
\mathbf{E}_{a l c, i}^{*}=\underset{\mathbf{E}_{a l c, i}}{\arg \max } \mathbf{1}^{T} \mathbf{U}_{i}
$$

$$
\begin{array}{ll}
\text { subject to } & \eta \mathbf{1}^{T} \mathbf{E}_{\text {har }, i}-\eta \mathbf{1}^{T} \mathbf{E}_{a l c, i, T_{D}}-\mathbf{1}^{T} \mathbf{E}_{a l c, i, T_{N}} \\
& -\mathbf{1}^{T} \mathbf{E}_{\text {leak }, i}=\Phi, \\
& \mathbf{E}_{\text {alc }, i} \succeq 0,
\end{array}
$$

where $\eta \mathbf{1}^{T} \mathbf{E}_{\text {har, } i}$ is the energy recharged with efficiency $\eta$ during the day, $\eta \mathbf{1}^{T} \mathbf{E}_{a l c, i, T_{D}}$ and $\mathbf{1}^{T} \mathbf{E}_{a l c, i, T_{N}}$ are the energy consumption for the daytime $T_{D}$ and the nighttime $T_{N} . \mathbf{1}^{T} \mathbf{E}_{\text {leak, } i}$ is the leakage energy during the day and $\Phi$ is a guard band to overcome estimation errors.

In particular, the first constraint is an affine function of $E_{a l c, i}[t]$ and the second constraint $\mathbf{E}_{a l c, i} \succeq 0$ is an intersection of half-spaces with respect to $E_{a l c, i}[t]$. Thus, the constraint set is convex. Since the objective function is the sum of concave 


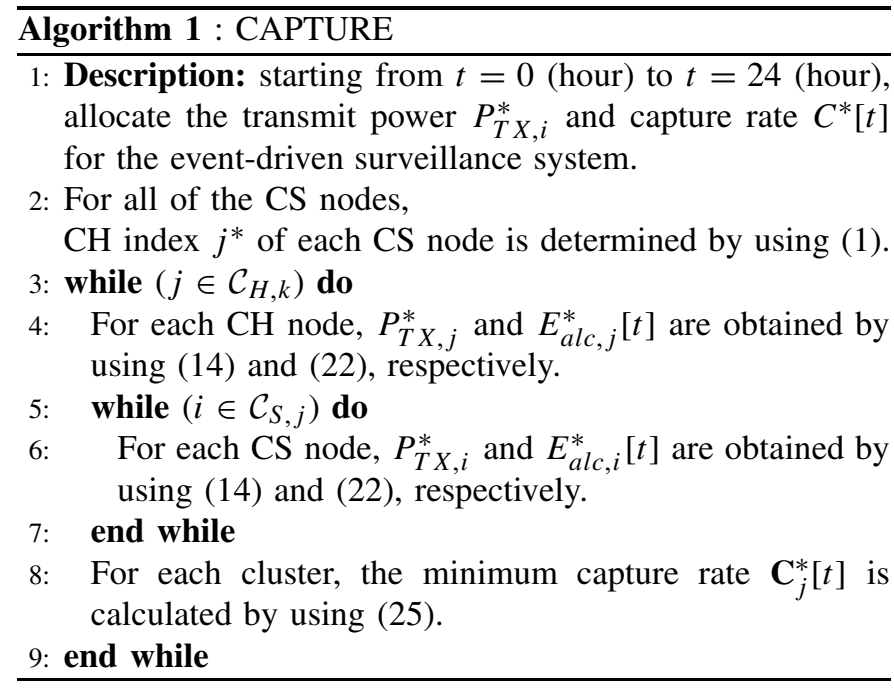

functions, it is also concave. Therefore, the optimization problem in (21) is convex with respect to $E_{a l c, i}[t]$, hence its karush-kuhn-tucker (KKT) conditions are necessary and sufficient for optimality.

By using the KKT condition, the optimal solution can then be obtained by $E_{a l c, i}^{*}[t]=\left[\frac{1}{e[t] v_{i}^{*}}-\frac{1}{w_{i, t}}\right]^{+}$where $e[t]=\eta$ for $t \in T_{D}$ and $e[t]=1$ for $t \in T_{N}$. By applying this to the constraint set,

$$
\begin{aligned}
& \sum_{t \in\left[t_{0}, t_{0}+n_{s c} \cdot \Delta t\right]} E_{a l c, i}^{*}[t] \\
= & \sum_{t \in\left[t_{0}, t_{0}+n_{s c} \cdot \Delta t\right]} \frac{1}{e[t]} \cdot\left[\frac{1}{v_{i}^{*}}-\frac{e[t]}{\overline{\mathbb{P}}_{a c t, S \| H}^{i}[t]}\right]^{+} \\
= & \sum_{t \in\left[t_{0}, t_{0}+n_{s c} \cdot \Delta t\right]} \frac{1}{e[t]} \cdot\left\{\eta E_{\text {har, }, i}[t]-E_{\text {leak }}[t]-\frac{\Phi}{n_{s c}+1}\right\} .
\end{aligned}
$$

$E_{a l c, i}^{*}[t]$ is always nonnegative. Thus, the allocated energy $E_{a l c, i}^{*}[t]$ becomes $\frac{1}{e[t]} \cdot\left[\frac{1}{v_{i}^{*}}-\frac{e[t]}{\overline{\mathbb{P}}_{a c t, S \| H}^{i}[t]}\right]^{+}$where $E_{a l c, i}^{*}[t]$ is proportional to the difference between the water level $\frac{1}{v^{*}}$ and the inverse weight $\frac{e[t]}{w_{i}[t]}=\frac{e[t]}{\overline{\mathbb{P}}_{a c t, S H H}^{i}[t]}$ [29]. In particular, the second and third equations of (22) imply that the waterlevel $\frac{1}{v_{i}^{*}}$ should be allocated according to the time $\left(T_{D}\right.$ or $\left.T_{N}\right)$ and the activation probability $\left(\overline{\mathbb{P}}_{a c t, S|| H}^{i}[t]\right)$ over $\eta E_{h a r, i}[t]-$ $E_{\text {leak }}[t]-\frac{\Phi}{n_{s c}+1}$ i.e., the total rechargeable energy $\eta E_{\text {har }, i}[t]$, excluding the leakage energy $E_{\text {leak }}[t]$ and the guard band energy $\frac{\Phi}{n_{s c}+1}$. As a result, the optimal energies $E_{a l c, i}^{*}[t]$ and $E_{a l c, j}^{*}[t]$ are allocated to all of the schemes in Eqs. (16) and (17) in Sec. III-C.

\section{Optimal Capture Rate}

In this work, we calculate the optimal capture rate of our system based on the allocated energy $\left(E_{a l c, i}^{*}[t], E_{a l c, j}^{*}[t]\right)$ and the required energy $\left(E_{\text {req }, i}[t], E_{r e q, j}[t]\right)$. By employing $E_{a l c, i}^{*}[t]$ and $E_{a l c, j}^{*}[t]$ instead of $E_{r e q, i}[t]$ and $E_{r e q, j}[t]$ in
Eq. (16) and (17) in Sec. III-C, it is possible to derive the optimal capture rate based on the optimal energy allocation of Eq. (21). Here, by classifying $E_{r e q, i}[t]$ of Eq. (16) into two parts : necessary energy consumption for the basic functions needed to maintain the EH-WVSN $\left(E_{n e c, i}=E_{m p}+E_{d e t}+\right.$ $\bar{E}_{L I N A, i}$, and the optional energy consumption for network operations at high levels of quality and capture rates $\left(E_{\text {opt }, i}=\right.$ $\left.\bar{E}_{B P, i}+\bar{E}_{C A P, i}+\bar{E}_{S I N A, i}-\bar{E}_{L I N A, i}\right), E_{r e q, i}[t]$ can be derived as follows

$$
E_{\text {alc,i}}^{*}[t] \geq \mathbf{C}_{i}[t] \cdot \widehat{E}_{r e q, i}[t] .
$$

where $\widehat{E}_{\text {req,i }}[t]$ is defined as the required energy per time $t$ for node $i$ which is $\widehat{E}_{r e q, i}[t]=\left(E_{\text {nec }, i}+\mathbb{P}_{a c t, S}^{i}[t] \cdot E_{\text {opt }, i}\right)$.

On the other hand, $E_{r e q, j}[t]$ of Eq. (24) can be classified into three parts, $\left(E_{n e c, j}=\bar{E}_{L I N A, j}\right),\left(E_{o p t 1, j}=E_{\text {wake }}+\right.$ $\left.E_{\text {stab }}+E_{\text {init }}+E_{\text {grab }}+E_{\text {rec }}+E_{\text {shut }}\right)$ and $\left(E_{\text {opt } 2, j}=\bar{E}_{B P, j}+\right.$ $\left.\bar{E}_{C A P, j}+\bar{E}_{C F P, j}+\bar{E}_{S I N A, j}-\bar{E}_{L I N A, j}\right)$. Thus, $E_{r e q, j}[t]$ becomes

$$
E_{a l c, j}^{*}[t] \geq \mathbf{C}_{j}[t] \cdot \widehat{E}_{r e q, j}[t] .
$$

where $\widehat{E}_{r e q, j}[t]$ is defined as the required energy per time $t$ for node $j$ which is $\widehat{E}_{\text {req }, j}[t]=\left(E_{\text {nec }, j}+\mathbb{P}_{\text {cap }, j}[t] \cdot E_{\text {opt } t, j}+\right.$ $\left.\mathbb{P}_{a c t, H}^{j}[t] \cdot E_{\text {opt } 2, j}\right)$.

Finally, since the optimal capture rate should be an integer value at the intersection of conditions (23) and (24) for each cluster, the optimal capture rate is obtained by

$$
\mathbf{C}_{j}^{*}[t]=\min \left\{\bigcup_{i \in \mathcal{C}_{S, j}}\left\lfloor\frac{E_{a l c, i}^{*}[t]}{\widehat{E}_{r e q, i}[t]}\right\rfloor,\left\lfloor\frac{E_{a l c, j}^{*}[t]}{\widehat{E}_{r e q, j}[t]}\right\rfloor\right\} .
$$

In other words, as shown in Eqs. (23) and (24), the integer upper bounds of the achievable capture rates determined for node $i$ and $j$ can vary with the allocated energy at each node $\left(E_{a l c, i}^{*}[t], E_{\text {alc }, j}^{*}[t]\right)$ and the required energy over time $t$ $\left(\widehat{E}_{r e q, i}[t], \widehat{E}_{r e q, j}[t]\right)$ but the optimal capture rate in Eqs. (23) and (24) should be the minimum value among the capture rates in each cluster involved in the EH-WVSN.

According to the pseudo codes of Algorithm 1, the capture algorithm determines the transmit power of each node and the synchronized capture rate of this system, as specified in Step 1. Towards this end, in Step 2, CS nodes are clustered with respect to a specific $\mathrm{CH}$ node by using (1). In the loop from Step 3 to Step 9, the optimal parameters for transmit power, allocated energy, and capture rate are determined by using (14), (22) and (25). The optimal allocated energy is updated per scheduling time $\Delta t$, but the transmit power is updated per day $(0 h-24 h)$. The main parameters of this paper are summarized in Table I.

\section{Simulation Results}

\section{A. Simulation Environments}

As shown in Fig. 6, three webcam nodes defined as $\mathrm{CH}$ nodes and ten CMOS camera nodes defined as the CS nodes were deployed between a server (embedded PC) and a parking lot in a surveillance scenario. Each node 


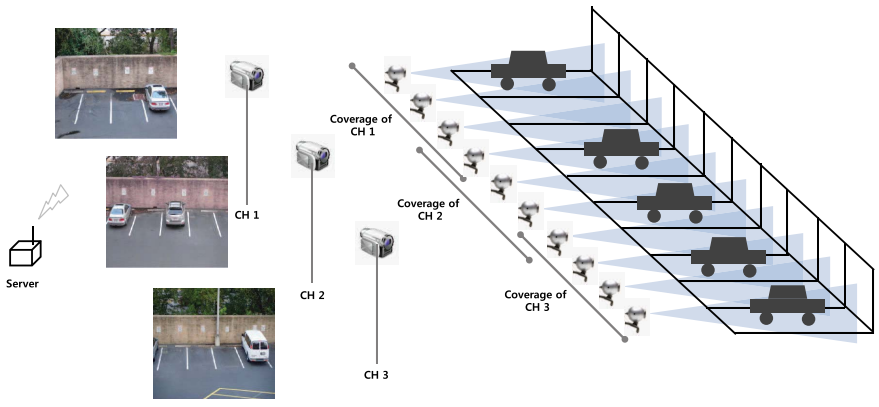

Fig. 6. Deployment of camera sensor nodes on a parking lot.

is assumed to be equipped with an energy harvesting module.

The CMOS camera nodes (CS node) capture the scenes with a periodic capture rate $\mathbf{C}[t]$. If an object or objects are detected, the CS nodes send a notification message to their $\mathrm{CH}$ nodes. Thus, the $\mathrm{CH}$ node captures scenes and recognizes whether any objects of interest are detected.

The harvesting energy pattern supplied by the solar panel is represented in Fig. 11(a) according to (15). Assume that $h_{\text {peak }}$ is $18 \mathrm{~mW} / \mathrm{cm}^{2}$ and $A$ is $100 \mathrm{~cm}^{2}$. In particular, $E_{\text {leak }}[t]$ should be determined experimentally [30]. However, since these parameters do not have a significant effect on performance, it is adequate to model it as a linear equation as a function of time, $E_{\text {leak }}\left[t_{0}+k \Delta t\right] \approx E_{\text {res }}\left[t_{0}\right] r_{\text {leak }} k \Delta t$, where $r_{\text {leak }}=0.01$ is the leakage ratio of the stored energy ( $\mathrm{J} / \mathrm{hour}$ ) and the maximum capacity of battery $B_{\max }$ is assumed to be large enough to recharge the solar energy. We also assume that the energy consumption for image processing in (16) and (17) is $E_{m p}=13.4 \mathrm{~mJ}, E_{\text {det }}=153.8 \mathrm{~mJ}, E_{\text {wake }}=368.9 \mathrm{~mJ}$, $E_{\text {stab }}=1161 \mathrm{~mJ}, E_{\text {init }}=1725.4 \mathrm{~mJ}, E_{\text {grab }}=537.2 \mathrm{~mJ}$, $E_{\text {rec }}=144.2 \mathrm{~mJ}$ and $E_{\text {shut }}=768.5 \mathrm{~mJ}$ [6].

In order to optimize the EH-WVSN using (22) and (25), we record the statistics of detection and recognition events in the parking lot of the Engineering Science Building at The University of Texas in Austin. The associated PDFs for object detection and recognition are represented in Fig. 7(a).

In our parking lot system, the position, orientation, and zoom level of each camera are fixed, so the $\operatorname{FoV}\left(\mathbf{X}_{i}, \mathbf{O}_{i}, Z_{i}\right)$ of node $i$ can be simplified as $F o V_{i}$. As shown in Fig. 7(b), we perform the clustering for visual sensor nodes over the environment in Fig. 6. When the FoVs of the nodes are assumed to be

$$
\begin{aligned}
& \left\{F o V_{n 1} \cup F_{o} V_{n 2} \cup F_{o} V_{n 3} \cup F_{o} V_{n 4}\right\} \subset F o V_{c h 1}, \\
& \left\{F o V_{n 4} \cup F_{o} V_{n 5} \cup F_{o} V_{n 6} \cup F_{o} V_{n 7}\right\} \subset F o V_{c h 2}, \\
& \left\{F o V_{n 7} \cup F_{o} V_{n 8} \cup F_{o} V_{n 9} \cup F_{o} V_{n 10}\right\} \subset F o V_{c h 3},
\end{aligned}
$$

their clusterings are composed of $\mathcal{C}_{S, c h 1}=\{n 1, n 2, n 3\}$, $\mathcal{C}_{S, c h 2}=\{n 4, n 5, n 6, n 7\}$, and $\mathcal{C}_{S, c h 3}=\{n 8, n 9, n 10\}$ according to Eq. (1) as shown in Fig. 7(b). Here, $n 4$ belongs to $c h 2$ but it can switch to $c h 1$ if the queue of $c h 1$ is shorter than the queue of $c h 2$. Likewise, $n 7$ can also switch to $c h 3$ to reduce the queueing delay.

The parameters used for the superframe control are arranged in Table II which are utilized for Eqs. (29), (30), (31) and (32).

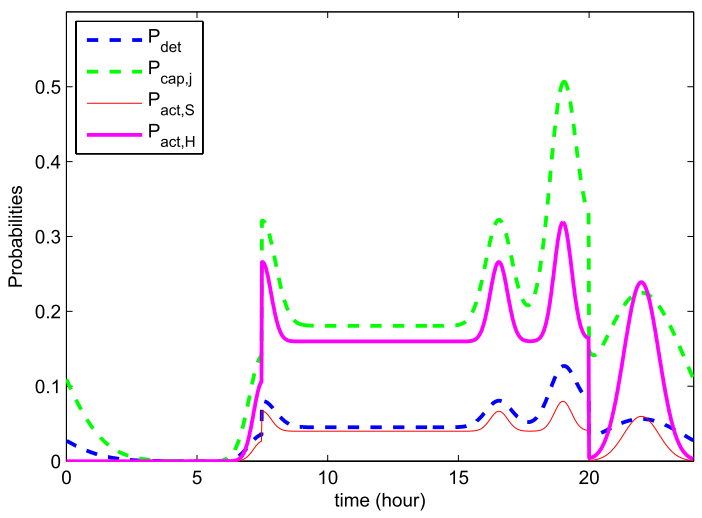

(a)

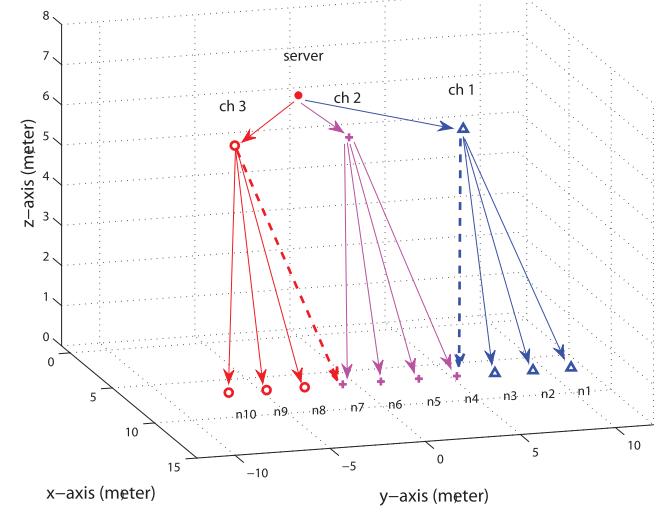

(b)

Fig. 7. System Environments. (a) Capture and activation probabilities. (b) Routing based on Clustering.

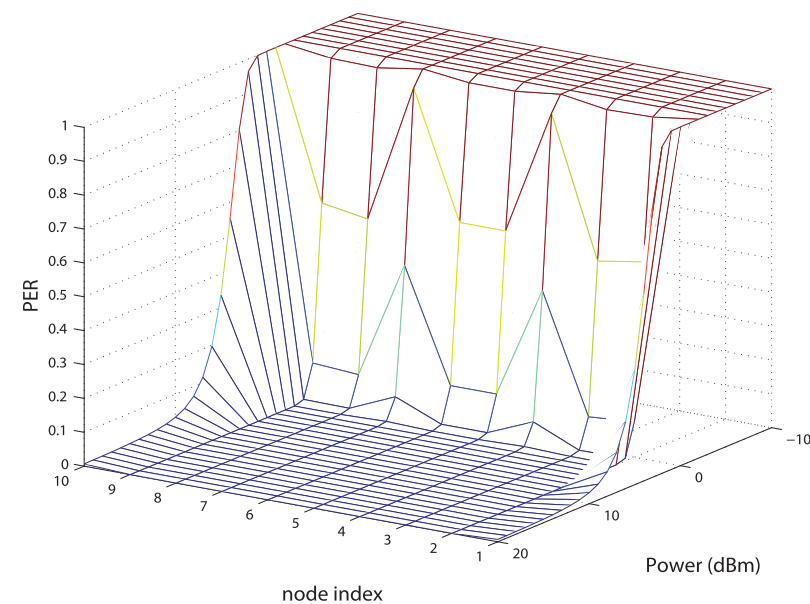

Fig. 8. Packet error rate of EH-WVSN.

The optimal transmit power for data transmission is calculated with Eq. (14). Fig. 8 shows the PER as a function of the node index (node position) over the range of transmit power, $-10 \mathrm{dBm} \leq P_{t x} \leq 20 \mathrm{dBm}$. In this simulation, we take into account the interference that occurs due to the hidden node problem and from utilizing the common mode power control, i.e., all the nodes use the same transmit power. As a result, the PER rapidly increases at $P_{t x}<3 \mathrm{dBm}$. In addition, nodes (n1 and n10) located at the border have a higher PER than the other nodes, since they suffer from more interference from hidden nodes and, due to the greater distance, their signals 
reach their $\mathrm{CHs}$ with weak strength relative to the other nodes (n2 or $\mathrm{n} 3$ ).

\section{B. Visual Surveillance Communication}

To simplify the analysis of image processing, intra-frame (I-frame) coding of H.264/AVC is employed for data reduction. At the initiation time, ch1 captures and encodes its FoV of Fig. 9(a) and enters into the sleep state until it receives a detection signal from one of the CS nodes in the cluster. Once ch1 gets the wake-up message from $n 3$ due to object detection as in Fig. 9(b), ch1 captures its FoV again and recognizes it as a car, then requests $\mathrm{n} 3$ to send the captured image of Fig. 9(b). Finally, ch1 transmits the aggregate and coded images of Fig. 9(b) and the difference image between Fig. 9(a) and Fig. 9(c) to the PAN coordinator. Likewise, all those procedures are applied to the other clusters as well. The sizes (340, 277.6, 322.2 Kbits) of the images in Fig. 9(c), 9(g), and 9(k) are reduced by subtraction to 191.2, 178.4 and 280 Kbits, respectively from each image size, as shown in Fig. 9(d), 9(h), and 9(1) at the next mini-slot $\tau_{0}+\Delta \tau$.

\section{Algorithm Description}

For performance comparison, two power allocation algorithms are employed as the benchmarks for the CAPTURE algorithm. One is an equal power allocation (EPA) algorithm and the other is the algorithm for the maximal sum of allocated power $(M S A P)$. The transmit power is optimally obtained by using Eq. (14).

1) EPA: Equal power is allocated to each CS node including the $\mathrm{CH}$ node during the day satisfying the following objective:

$$
\begin{aligned}
\underset{E_{\text {alc }, i}^{*}}{\arg \min }\left\{E_{R}=\eta \cdot \sum_{t \in T}\left[E_{\text {har }, i}[t]-E_{\text {alc }, i}^{*}\right]^{+}\right\}, \\
\text {s.t. } E_{R} \geq \sum_{t \in T}\left[E_{\text {alc }, i}^{*}-E_{\text {har }, i}[t]\right]^{+}, \text {for } i \in\left(\mathcal{C}_{S, j}, j\right)
\end{aligned}
$$

where $E_{R}$ is the recharged energy, $\eta$ is the recharged efficiency into the battery and $T$ is the scheduling time set during the day. From this algorithm, an equal optimal power is determined to be drained of the harvesting energy during the day.

2) MSAP: The power allocation is performed for each CS node including the $\mathrm{CH}$ node to guarantee a lower bound of capture rate with reference to a threshold of power. After guaranteeing the required power, we additionally allocate the residual harvesting energy to the node. The allocated energy is determined during the scheduling time $t$ for $i \in\left(\mathcal{C}_{S, j}, j\right)$

$$
E_{\text {alc }, i}^{*}[t]=\left\{\begin{array}{lc}
E_{\text {alc }, i}^{\min *} & \text { if } t \in T_{S N} \\
\min \left(E_{\text {har }, i}[t], E_{\text {alc }, i}^{\max }\right) & \text { else }\left(t \in T_{S D}\right)
\end{array}\right.
$$

where $E_{a l c, i}^{\min *}$ is the energy bound guaranteeing the lower bound of capture rate, $E_{a l c, i}^{\max *}$ is a maximal energy bound determined by means of the residual harvesting energy, $T_{S N}=\left\{t \mid E_{\text {har }, i}[t]-E_{\text {alc }, i}^{\text {min }}{ }^{*}<0\right\}$ and $T_{S D}=\left\{t \mid E_{\text {har }, i}[t]-\right.$ $\left.E_{\text {alc }, i}^{\min *}>0\right\}$.

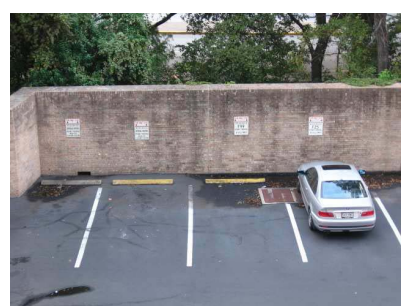

(a)

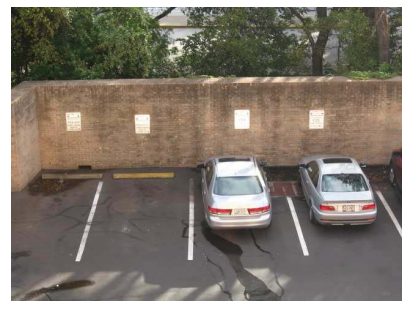

(c)

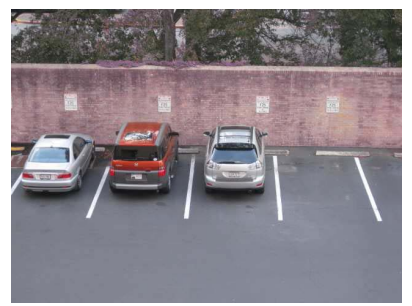

(e)

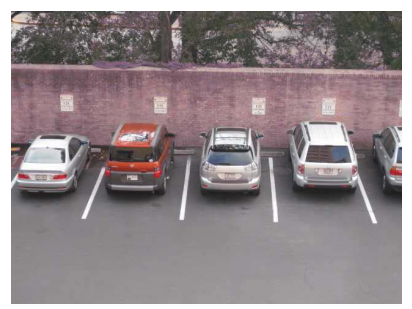

(g)

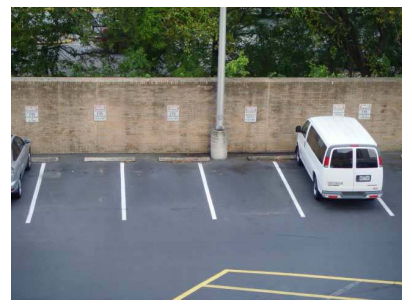

(i)

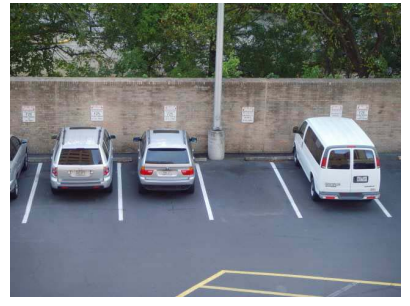

(k)

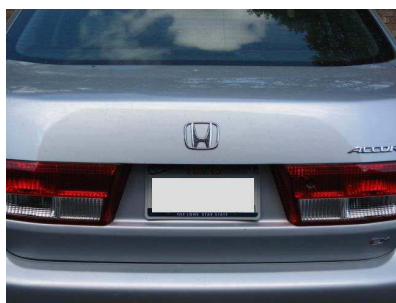

(b)

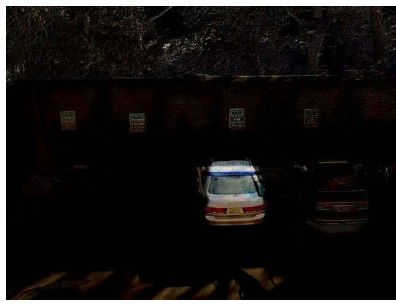

(d)

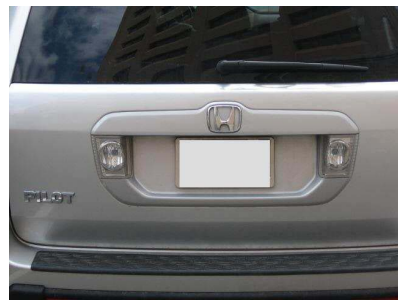

(f)

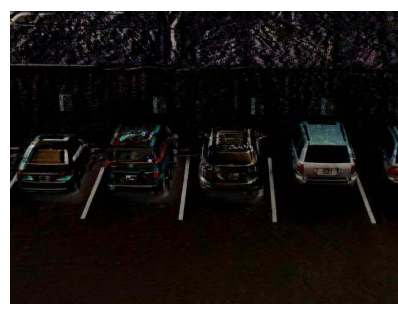

(h)

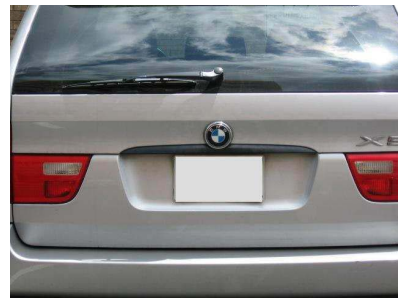

(j)

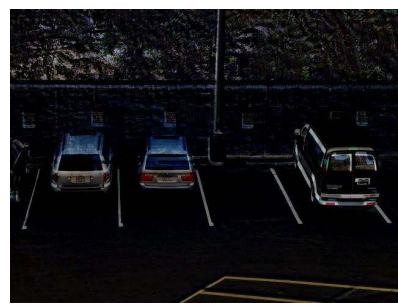

(1)
Fig. 9. Images captured at the $1^{s t}$ tier nodes and the $2^{\text {nd }}$ tier nodes. (a) FoV (ch1) at $\tau_{0}$. (b) FoV (n3) at $\tau_{1}$. (c) FoV (ch1) at $\tau_{1}$. (d) Difference image of (a) and (c). (e) FoV (ch2) at $\tau_{0}$. (f) FoV (n7) at $\tau_{1}$. (g) FoV (ch2) at $\tau_{1}$. (h) Difference image of (e) and (g). (i) FoV (ch3) at $\tau_{0}$. (j) FoV (n8) at $\tau_{1}$. (k) FoV (ch3) at $\tau_{1}$. (1) Difference image of (i) and (k).

3) Capture: The power is allocated to each node including the $\mathrm{CH}$ node based on the number of generated events at each 


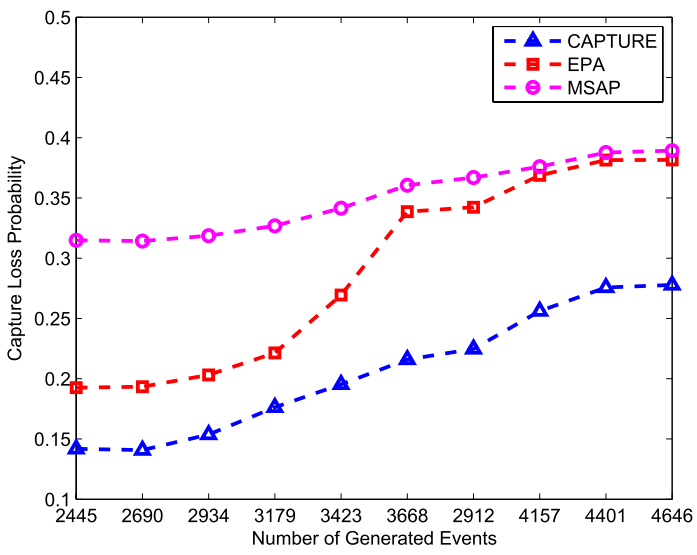

(a)

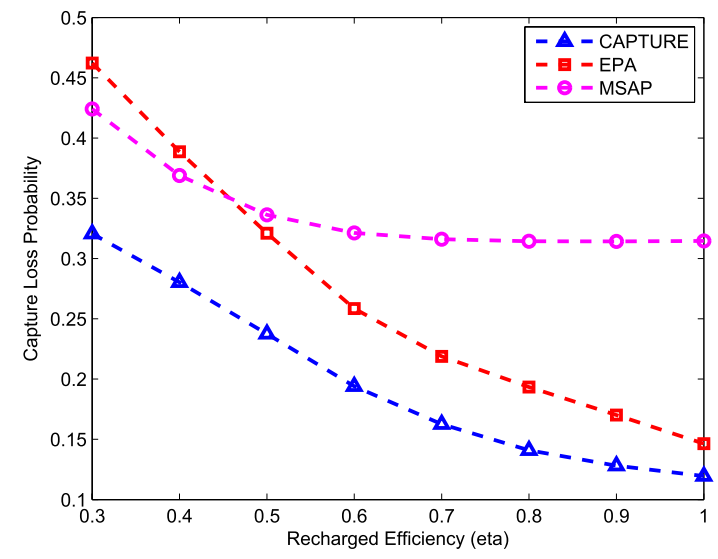

(b)

Fig. 10. Capture loss probabilities at CS node. (a) According to the average generated event (per day). (b) According to the recharged battery efficiency.

scheduling time. Different from EPA and MSAP, CAPTURE reflects the statistics of the generated events so that the patterns of the allocated energies $\left(E_{\text {alc }, S}^{*}[t], E_{\text {alc }, H}^{*}[t]\right)$ follow the activation probabilities $\left(\overline{\mathbb{P}}_{a c t, S}[t], \overline{\mathbb{P}}_{a c t, H}[t]\right)$ as described in Algorithm 1.

\section{Experimental Analysis}

Fig. 10(a) shows the capture loss probability according to the number of generated events, which is the average value after performing 100 iterations for performance reliability. The probability for each algorithm monotonically increases when the number of generated events is larger. The probability for CAPTURE is relatively lower than the other two algorithms, which stems from reflecting the temporal statistics of the generated events. Fig. 10(b) depicts the probability according to the recharged efficiency $\eta$ where CAPTURE exhibits a lower probability than the other two algorithms since it allocates the harvesting energy based on the event activation probability. In addition, the capture loss probability is reduced for EPA when the recharged efficiency $\eta$ approaches one. By contrast, in MSAP, it is not improved much even if the recharged efficiency $\eta$ is larger. The EPA is more efficient than the allocation to have a maximal sum of power when the recharged efficiency $\eta$ is one.

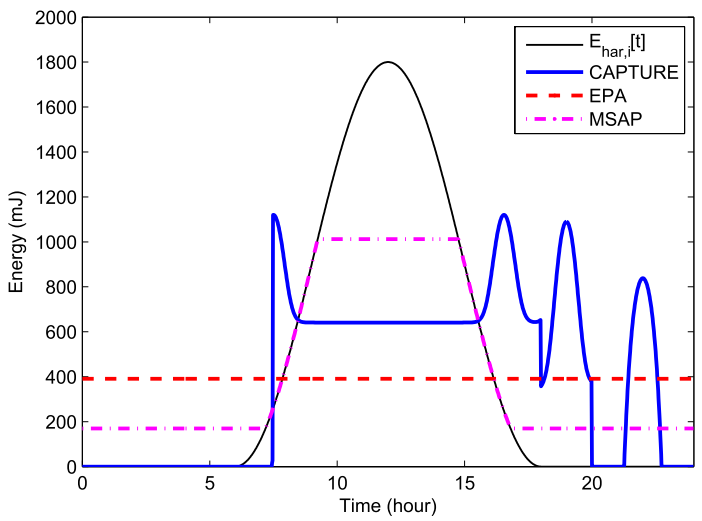

(a)

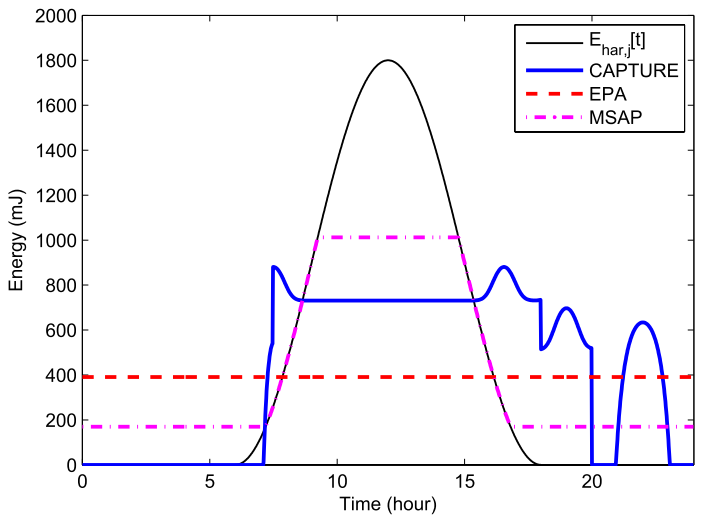

(b)

Fig. 11. Optimal energy allocation. (a) Allocated energy at CS. (b) Allocated energy at $\mathrm{CH}$.

Figs. 11 and 12 show the allocated energies and capture rates for each node including the $\mathrm{CH}$ node using Eqs. (22), (23), and (24). The number of generated events is 2445 and the recharged efficiency $\eta$ is 0.8 . It is shown that an equal power is allocated. MSAP allocates the power in order to guarantee the lower bound of the capture rate. The residual harvesting energy is then allocated without recharging it. the lower bound of capture rate, the residual harvesting energy is allocated to the node as shown in Fig. 11. On the other hand, by comparing Fig. 7(a) and Fig. 11, it can be seen that the allocation of CAPTURE reflects the statistics of the generated events.

During $16 \sim 19$ hours, people go home after work while other people arrive in the lot for night work. Due to the movement of people as well as the traffic flow, the capture probability during these hours is very high, because both automobiles and other objects are in motion. Hence the activation probability due to false-alarm is relatively small.

From Fig. 12, it can be seen that the average capture rate is approximately 4 per minute, which is usually good enough for parking lot surveillance but insufficient for real-time surveillance, due to the limited amount of harvesting energy and the large volume of image data.

However, the achievable capture rate at the $\mathrm{CH}$ in Fig. 12(b) using (24) is much higher than the result in Fig. 12(a) using (23), since the required energy of the $\mathrm{CH}, \widehat{E}_{r e q, j}[t]$, is much 


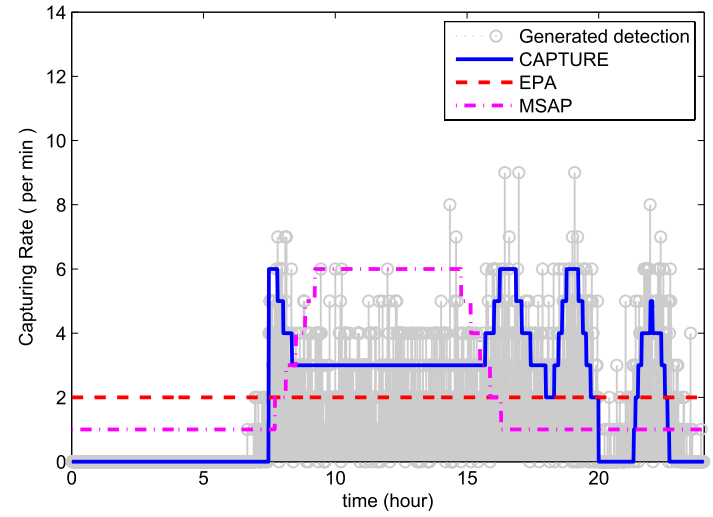

(a)

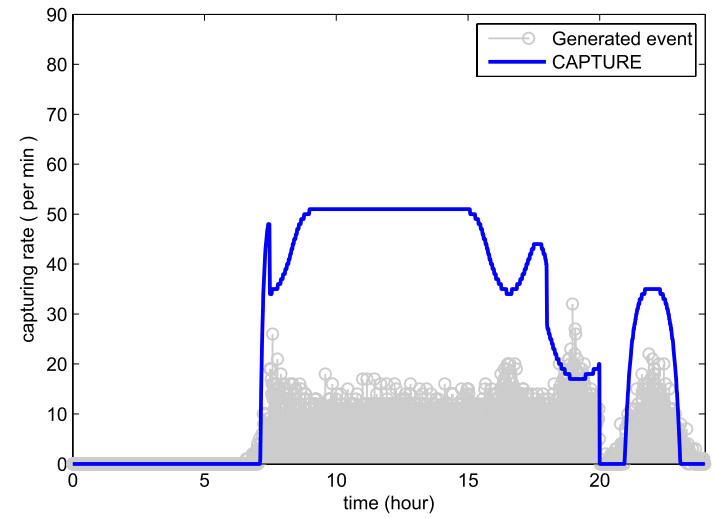

(b)

Fig. 12. Optimal capture rate. (a) Optimal capture rate at CS. (b) Achievable capture rate at $\mathrm{CH}$.

smaller than that of the CS node, $\widehat{E}_{r e q, i}[t]$, i.e. $E_{a l c, i}^{*}[t] \approx$ $E_{\text {alc }, j}^{*}[t]$ but $\widehat{E}_{r e q, i}[t] \gg \widehat{E}_{r e q, j}[t]$ in $\mathbf{C}_{i}[t]=\frac{E_{\text {alc },}^{*}[t]}{\widehat{E}_{\text {req }, i}[t]} \ll$ $\frac{E_{a l c, j}^{*}[t]}{\bar{E}_{r e q, j}[t]}=\mathbf{C}_{j}[t]$ where $\widehat{E}_{r e q, j}$ is multiplied by the small probability $\mathbb{P}_{\text {cap }}[t]$.

Therefore, the major factor limiting the performance of a VSN is not the energy needed for communication, but the energy needed for accomplishing image processing tasks such as capture, detection and recognition, which is quite different from conventional scalar sensor networks [31], [32] and from studies of theoretical visual sensor networks [20]. Although it is inevitable that a large amount of energy is used for image processing, it is possible to control this through capture rate control.

Fig. 13 shows the valid capture rate for the detected event according to the transmit energy per capture energy at the CS node. For Case I, we use the probability patterns in Fig. 7(a) for the activation probability of the CS node. For Case II, the probability of the CS node is three times more than that of Fig. 7(a). Thus, Case II has a larger number of generated events three times more than Case I. If the transmit energy increases, conversely, the available energy for capturing events decreases. Fig. 13 shows the capture rate decreasing when the transmit energy per capture energy is increasing. Since Case II has more activations, it can be seen that the

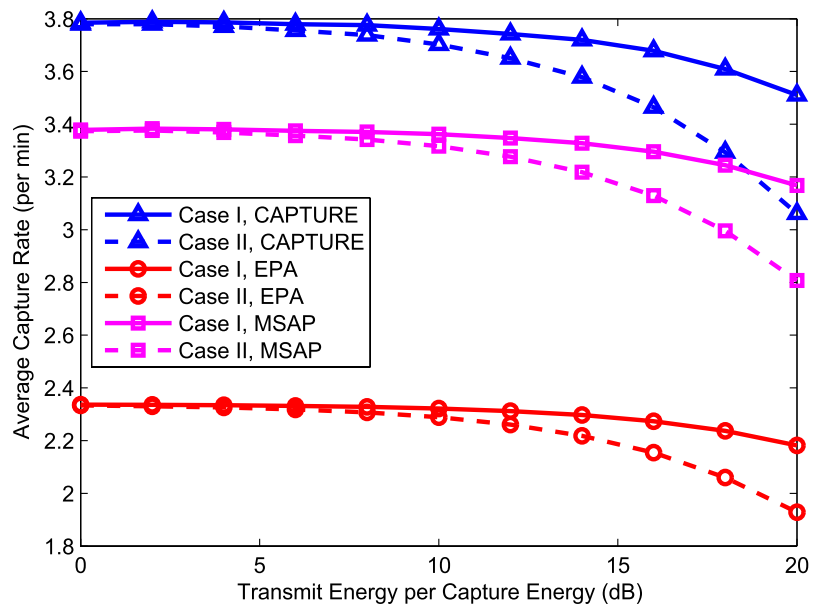

Fig. 13. Relation between the transmit energy and capturing rate at CS node.

capture rate is steeply decreases when the transmit energy per capture energy is over 15. In particular, CAPTURE determines the capture rate based on the statistics of the generated events so that it can save the harvesting energy during the idle state; this leads to a higher average capture rate than the others.

\section{CONCLUSION}

This paper studied the optimal system configurations of EH-WVSNs with regard to energy allocation and capture rate control. We presented a unique algorithm called CAPTURE that is suitable for handling 2-D and 3-D visually sensed information (video). We attempt to find a new approach to the optimization of EH-WVSNs by exploring a new framework that describes the energy harvesting system. As a practical simulation, we demonstrated the developed networking methodologies by using the IEEE 802.15.4 superframe structure which lead to enhanced lifetimes of the WVSN nodes. In order to obtain an optimal capture rate over the EH-WVSN, we derived an optimal solution using event detection and recognition probabilities and energy flow constraints. In the simulations, we demonstrated the feasibility of the proposed EH-WVSN approach in terms of energy consumption, energy allocation and capture rate with a realistic scenario "parking lot surveillance" and analyzed the relationship between the network operation and the capture operation.

\section{APPENDIX I}

Time Duration AND ENERGY CONSUMPTION OF SHORT INACTIVE PERIOD, BP, CAP AND CFP

\section{A. Inactive Period}

When $\mathbb{P}_{a c t}=1, \bar{t}_{\text {sup }}$, and $\bar{t}_{B P}$ are fixed and $\bar{t}_{S I N A}$ varies dependently with $\bar{t}_{C A P}$ and $\bar{t}_{C F P}$. Therefore, $\bar{E}_{S I N A}$ is given by

$$
\bar{E}_{S I N A, S}^{i}=\bar{t}_{S I N A, S}^{i} \cdot P_{S}, \quad \bar{E}_{S I N A, j}=\bar{t}_{S I N A, H}^{j} \cdot P_{S},
$$


TABLE II

NOTATIONS USED FOR THE ANALYSIS OF IEEE 802.15.4

\begin{tabular}{||c|c|c||c|c|c||}
\hline Notation & Meaning & Value & Notation & Meaning & Lalue \\
\hline$t_{S I F S}$ & SIFS & $192 \mu \mathrm{S}$ & $t_{L I F S}$ & $640 \mu \mathrm{S}$ \\
\hline$t_{S I}$ & Transient time (sleep to idle) & $970 \mu \mathrm{S}$ & $t_{I T}$ & Transient time (idle to transmit) & $192 \mu \mathrm{S}$ \\
\hline$t_{T R}$ & Transient time (transmit to receive) & $200 \mu \mathrm{S}$ & $t_{R T}$ & Transient time (receive to transmit) & $220 \mu \mathrm{S}$ \\
\hline$t_{I R}$ & Transient time (idle to receive) & $192 \mu \mathrm{S}$ & $t_{S L}$ & Time duration of sleep state & variable \\
\hline$t_{A W}$ & ACK wait duration & $864 \mu \mathrm{S}$ & $R$ & Radio rate & $250 \mathrm{Kbps}$ \\
\hline$L_{L}$ & LDF length & $127 \mathrm{~B}$ & $L_{B}$ & Beacon frame length & $26 \mathrm{~B}$ \\
\hline$L_{A}$ & ACK frame length & $11 \mathrm{~B}$ & $L_{S}$ & SDF (Short Data Frame) length & 18 B \\
\hline$t_{B O T}$ & Backoff time & Variable & $E_{B O T}$ & Backoff energy & Variable \\
\hline$P_{I}$ & Power consumption for idle state & $2.79 \mathrm{~mW}$ & $P_{S}$ & Power consumption for sleep state & $30 \mu \mathrm{W}$ \\
\hline$P_{T X}$ & Transmit power & variable & $P_{R X}$ & Receive power & $56.5 \mathrm{~mW}$ \\
\hline
\end{tabular}

where $P_{S}$ is the power consumption for the sleep state and

$$
\begin{aligned}
\bar{t}_{S I N A, S}^{i}= & \bar{t}_{\text {sup }, S}^{i}\left(\mathbb{P}_{a c t, S}^{i}=1\right)-\bar{t}_{B P, S}^{i} \\
& -t_{C A P, S}^{i}-\sum_{x \in \mathcal{C}_{S, j} \backslash\{i\}} \mathbb{P}_{a c t, S}^{x} \cdot t_{C A P, S}^{x}, \\
\bar{t}_{S I N A, H}^{j}= & \bar{t}_{\text {sup }, H}^{j}\left(\mathbb{P}_{a c t, H}^{j}=1\right)-\bar{t}_{B P, H}^{j} \\
& -\sum_{y \in \mathcal{C}_{H, k} \backslash\{j\}} \sum_{n^{\prime} \subset \mathcal{C}_{S, y}}\left[\prod_{i^{\prime} \in n^{\prime}} \mathbb{P}_{a c t, S}^{i^{\prime}}\right. \\
& \left.\cdot \prod_{l^{\prime} \in \mathcal{C}_{S, y} \backslash n^{\prime}}\left\{1-\mathbb{P}_{a c t, S}^{l^{\prime}}\right\} t_{C F P, H}^{y, n^{\prime}}\right] \\
& -\sum_{n \subset \mathcal{C}_{S, j}}\left[\prod_{i \in n} \mathbb{P}_{a c t, S}^{i} \cdot \prod_{l \in \mathcal{C}_{S, j} \backslash n}\left\{1-\mathbb{P}_{a c t, S}^{l}\right\}\right. \\
& \left.\cdot\left\{t_{C A P, H}^{n}-t_{C F P, H}^{j, n}\right\}\right] .
\end{aligned}
$$

\section{B. Average Length $\bar{t}_{B P}$ and Average Energy Consumption $\bar{E}_{B P}$ During BP}

The beacon duration and the energy consumption at the CS and $\mathrm{CH}$ nodes should be calculated based on the topology, i.e. only receive at the $\mathrm{CS}$ nodes and receive and transmit at the $\mathrm{CH}$ nodes.

$$
\begin{aligned}
& \bar{t}_{B P, S}^{i}=t_{R X B}, \bar{t}_{B P, H}^{j}=t_{R X B}+t_{T X B}, \\
& \bar{E}_{B P, S}^{i}=E_{R X B}, \bar{E}_{B P, H}^{j}=E_{R X B}+E_{T X B} .
\end{aligned}
$$

where $t_{R X B}$ and $E_{R X B}$ are the time duration and the energy required for beacon reception.

\section{Average Length $\bar{t}_{C A P}$ and Average Energy Consumption $\bar{E}_{C A P}$ During $C A P$}

Based on the principles of CSMA-CA operation, the CAP durations and the associated energy consumptions at node $i$ and $j$ can be calculated by

$$
\begin{aligned}
t_{C A P, S}^{i} & =\left(t_{T X D S}+t_{R X A}\right) \cdot u \cdot N_{F, i}, \\
E_{C A P, S}^{i} & =\left(E_{T X D S}+E_{R X A}\right) \cdot u \cdot N_{F, i}, \\
t_{C A P, S}^{x} & =\left(t_{T X D S}+t_{R X A}\right) \cdot u \cdot N_{F, x}, \\
E_{C A P, S}^{x} & =t_{C A P, S}^{x} \cdot P_{I}, \quad(x \neq i), \\
t_{C A P, H}^{n} & =\sum_{x \in n}\left(t_{R X D S}+t_{T X A}\right) \cdot u \cdot N_{F, x}, \\
E_{C A P, H}^{n} & =\sum_{x \in n}\left(E_{R X D S}+E_{T X A}\right) \cdot u \cdot N_{F, x},
\end{aligned}
$$

where $u$ is the average number of retransmissions. The terms $t_{T X D S}$ and $E_{T X D S}$ are the time duration and the energy for a short data transmission, $t_{R X A}$ and $E_{R X A}$ are the time duration and the energy for ACK reception. $P_{I}$ is the power consumption in the idle state, and $N_{F, i}$ is the total number of data frames after fragmenting the image captured at node $i$, $N_{F, i}=\left\lceil\frac{L_{i}}{L_{S}}\right\rceil$ where $L_{i}$ is the image size coded following capture at node $i$. Likewise, $N_{F, x}$ is the total number of frames after fragmenting the image captured at node $x, N_{F, x}=\left\lceil\frac{L_{x}}{L_{S}}\right\rceil$. Then, according to Table II,

$$
\begin{aligned}
t_{R X D S} & =t_{S I}+t_{B O T}+t_{I R}+\frac{L_{S}}{R}+t_{S I F S}, \\
E_{R X D S} & =\left(t_{R X D S}-t_{S I F S}\right) P_{R X}+t_{S I F S} P_{I} \\
t_{T X A} & =t_{R T}+\frac{t_{A W}}{2}+\frac{L_{A}}{R}, \\
E_{T X A} & =\left(t_{R T}+\frac{L_{A}}{R}\right) P_{T X}+\frac{t_{A W}}{2} P_{I} .
\end{aligned}
$$

\section{Average Duration $\bar{t}_{C F P}$ and Average Energy Consumption $\bar{E}_{C F P}$ During CFP}

The average duration $t_{C F P, j}^{j, n}$ and the average energy consumption $E_{C F P, j}^{j, n}$ over a CFP are computed next. Let $t_{\text {unit }}$ be the unit duration in a superframe $t_{\text {unit }}=\frac{S D}{16}=t_{B A S E} \cdot 2^{S O-4}$. Suppose node $j$ obtain a guaranteed time slot (GTS) from node $k$. In this case, let $n_{G T S, j}$ be the number of unit durations over the GTS. Then, the GTS duration becomes $t_{G T S, j}=n_{G T S, j} \cdot t_{\text {unit }}$. For example, Fig. 4 shows the GTS allocation for the transmission of images received from the node set $n$ and the coded image captured by node $j$ when $t_{G T S, j}=2 \cdot t_{\text {unit }}$. Here we assume that the GTS is occupied first by the combination of long data frame (LDF) and LIFS for maximum time utilization and then by the combination of 
left data frame and IFS: long inter frame spacing (LIFS) or short inter frame spacing (SIFS). The transmit duration of the LDFs is denoted by $t_{L}$, the duration for the LIFS by $t_{L I F S}$, the transmit duration of left by $t_{L E F T}$ and the duration for the IFS by $t_{I F S}$. This can vary with the length of the left data frame.

Likewise, the bit lengths of $t_{L}$ and $t_{L E F T}$ are denoted as $L_{L}$ and $L_{L E F T}$. In addition, $L_{L}$ is usually set to be $L_{L}=$ aMaxPHYPacketSize in [27]. The average duration and energy consumption for the GTS allocation become

$$
\begin{aligned}
t_{C F P, H}^{j, n}= & k_{j} \cdot t_{\text {unit }}=\left(N_{F, j}^{n}-1\right)\left[t_{L}+t_{L I F S}\right] \\
& +t_{L E F T, j}+t_{I F S}\left(L_{L E F T, j}\right)+t_{I D, j} \\
E_{C F P, H}^{j, n}= & \left(N_{F, j}^{n}-1\right)\left[E_{L}+E_{L I F S}\right] \\
& +E_{L E F T, j}+E_{I F S}\left(L_{L E F T, j}\right)+t_{I D, j} P_{I}
\end{aligned}
$$

where $E_{L}, E_{L I F S}, E_{L E F T, j}$ and $E_{I F S}$ are the energy consumptions during $t_{L}, t_{L I F S}, t_{L E F T, j}$ and $t_{I F S}$, respectively. Here $t_{I D, j}$ is the time duration of the idle state. In particular, $t_{I F S}$ and $E_{I F S}$ can differ according to $L_{L E F T}$ after allocating the LDFs,

$$
E_{I F S}=t_{I F S} \cdot P_{I}, \quad t_{I F S}= \begin{cases}t_{S I F S}, & \text { if } L_{L E F T, j} \leq L_{S} \\ t_{L I F S}, & \text { if } L_{L E F T, j}>L_{S} .\end{cases}
$$

In addition, $N_{F, j}^{n}$ is the total number of frames fragmented in the GTS duration $T_{G T S, j}$,

$$
N_{F, j}^{n}=\left\lceil\frac{L_{j}+\sum_{i \in n} L_{i}}{L_{L}}\right\rceil \leq\left\lceil\frac{t_{G T S, j}}{t_{L}+t_{L I F S}}\right\rceil,
$$

$L_{j}$ is the size (bits) of the coded image captured by node $j$ and $L_{G T S, j}$ is the GTS length (bits) allocated to node $j$. In addition, the residual data length $\left(L_{L E F T, j}\right)$ after allocating data frames of $N_{F, j}^{n}$ can be represented as $L_{L E F T, j}=\left(L_{j}+\right.$ $\left.\sum_{i \in n} L_{i}\right)-L_{L}\left(N_{F, j}^{n}-1\right)$. Then, $E_{L}$ and $E_{L E F T}$ are given by $E_{L}=t_{S I} P_{I}+\left(t_{I T}+\frac{L_{L}}{R}\right) P_{T X}, E_{L E F T}=t_{S I} P_{I}+\left(t_{I T}+\right.$ $\left.\frac{L_{L E F T, j}}{R}\right) P_{T X}$. In addition, $E_{L I F S}$ and $E_{S I F S}$ are given by $E_{L I F S}=t_{L I F S} P_{I}$ and $E_{S I F S}=t_{S I F S} P_{I}$. Lastly, $t_{I D, j}$ is

$$
\begin{aligned}
t_{I D, j}= & t_{G T S, j}-\left\{\left(N_{F, j}^{n}-1\right)\left[t_{L}+t_{L I F S}\right]\right. \\
& \left.+t_{L E F T, j}+t_{I F S}\left(L_{L E F T, j}\right)\right\} .
\end{aligned}
$$

On the other hand, by utilizing the same principle, $t_{C F P, H}^{y, n^{\prime}}$ and $E_{C F P, H}^{y, n^{\prime}}$ can be derived by

$$
\begin{aligned}
t_{C F P, H}^{y, n^{\prime}}= & k_{y} \cdot t_{\text {unit }}=\left(N_{F, y}^{n^{\prime}}-1\right)\left[t_{L}+t_{L I F S}\right] \\
& +t_{L E F T, y}+t_{I F S}\left(L_{L E F T, y}\right)+t_{S L, y}, \\
E_{C F P, H}^{y, n^{\prime}}= & \left(N_{F, y}^{n^{\prime}}-1\right)\left[E_{L}+E_{L I F S}\right] \\
& +E_{L E F T, y}+E_{I F S}\left(L_{L E F T, y}\right)+t_{S L, y} P_{I} .
\end{aligned}
$$

\section{REFERENCES}

[1] T. D. C. Little, J. Konrad, and P. Ishwar, "A wireless video sensor network for autonomous coastal sensing," in Proc. CESN, Apr. 2007.

[2] Y. Ma, S. Soatto, J. Kosecka, and S. S. Sastry, An Invitation to 3-D Vision: From Images to Geometric Models. New York, NY, USA: Springer-Verlag, 2003.

[3] L. Merino, J. Wiklund, F. Caballero, A. Moe, J. R. M. De Dios, P.-E. Forssen, et al., "Vision-based multi-UAV position estimation," IEEE Robot. Autom. Mag., vol. 13, no. 3, pp. 53-62, Sep. 2006.
[4] Z. Zivkovic and R. Kleihorst, "Smart cameras for wireless camera networks: Architecture overview," in Multi-Camera Networks: Concepts and Applications, A. Cavallaro and H. Aghajan, Eds. Amsterdam, The Netherlands: Elsevier, 2009, pp. 497-511.

[5] T. He, S. Krishnamurthy, J. A. Stankovic, and T. Abdelzaher, "Energy-efficient surveillance system using wireless sensor networks," in Proc. 2nd Int. Conf. Mobile Syst., Appl., Services, Jun. 2006, pp. $270-283$.

[6] P. Kulkarni, D. Ganesan, P. Shenoy, Q. Lu, and P.-E. Forssen, "SensEye: A multi-tier camera sensor network," in Proc. 13th Annu. ACM Int. Conf. Multimedia, Nov. 2005, pp. 229-238.

[7] I. F. Akyildiz, T. Melodia, K. R. Chowdhury, K. Nordberg, and A. Ollero, "A survey on wireless multimedia sensor networks," Comput. Netw., vol. 51, no. 4, pp. 921-960, Mar. 2007.

[8] I. F. Akyildiz, T. Melodia, and K. R. Chowdhury, "Wireless multimedia sensor networks: Applications and testbeds," Proc. IEEE, vol. 96, no. 10, pp. $1588-1605$, Oct. 2008.

[9] S. Misra, M. Reisslein, and G. Xue, "A survey of multimedia streaming in wireless sensor networks," IEEE Commun. Surv. Tuts. vol. 10, no. 4 , pp. 18-39, Oct./Dec. 2008.

[10] S. Soro and W. Heinzelman, "A survey of visual sensor networks," Adv. Multimedia, vol. 2009, pp. 640386-1-640386-21, May 2009.

[11] W. Feng, B. Code, E. Kaiser, M. Shea, W. Feng, and L. Bavoil, "Panoptes: A scalable architecture for video sensor networking applications," in Proc. ACM Multimedia, 2003, pp. 151-167.

[12] R. Kleihorst, B. Schueler, A. Danilin, and M. Heijligers, "Smart camera mote with high performance vision system," in Proc. ACM Workshop DSC, Oct. 2006.

[13] E. Ljung, E. Simmons, A. Danilin, R. Kleihorst, and B. Schueler, "802.15.4 powered distributed wireless smart cameras network," in Proc. Workshop DSC, Oct. 2006.

[14] R. Kleihorst, B. Schueler, and A. Danilin, "Solar power system design consideration," in Solar Power in Building Design. New York, NY, USA: McGraw-Hill, 2007, ch. 3.

[15] A. A. Abbo, R. P. Kleihorst, and B. Schueler, "Xetal-II: A low-power massively-parallel processor for video scene analysis," J. Signal Process. Syst., vol. 62, no. 1, pp. 17-27, Jan. 2011.

[16] P. Gevorkian, "Xetal-II: A low-power massively-parallel processor for video scene analysis," J. Signal Process. Syst., vol. 62, no. 1, pp. 17-27, Jan. 2011.

[17] K. Lin, J. Yu, J. Hsu, S. Zahedi, and D. Lee, "Heliomote: Enabling long-lived sensor networks through solar energy harvesting," in Proc. 3rd Int. Conf. Embedded Netw. Sensor Syst., Nov. 2005, p. 309.

[18] V. Raghunathan and P. H. Chou, "Design and power management of energy harvesting embedded systems," in Proc. ISLPED, Oct. 2006, pp. $369-374$.

[19] A. Kansal, S. Hsu, S. Zahedi, and M. Srivastava, "Power management in energy harvesting sensor networks," ACM Trans. Embedded Comput. Syst., vol. 6, no. 4, pp. 32-69, Sep. 2007.

[20] J. C. Dagher, M. W. Marcellin, and M. A. Neifeld, "A method for coordinating the distributed transmission of imagery," IEEE Trans. Image Process., vol. 15, no. 7, pp. 1705-1717, Jul. 2006.

[21] X. Gu, Z. Wen, C. Lin, and P. S. Yu, "ViCo: An adaptive distributed video correlation system," in Proc. 14th Annu. ACM Int. Conf. Multimedia, Oct 2006, pp. 559-568.

[22] F. Qureshi and D. Terzopoulos, "Distributed coalition formation in visual sensor networks: A virtual vision approach," in Proc. 3rd IEEE Int. Conf. Distrib. Comput. Sensor Syst., Jun. 2007, pp. 1-20.

[23] K.-Y. Chow, K.-S. Lui, and E. Lam, "Efficient on-demand image transmission in visual sensor networks," EURASIP J. Adv. Signal Process., vol. 2007 , no. 1, p. 225, Jan. 2007

[24] S. Bi, A. T. Kamal, C. Soto, D. Chong, J. A. Farrell, and A. K. Roy-Chowdhury, "Tracking and activity recognition through consensus in distributed camera networks," IEEE Trans. Image Process. vol. 19 , no. 10 , pp. $2564-2579$, Oct. 2010.

[25] R. Vidal, S. Rashid, C. Sharp, O. Shakernia, K. Jin, and S. Sastry, "Pursuit-evasion games with unmanned ground and aerial vehicles," in Proc. IEEE ICRA, vol. 3. Mar. 2001, pp. 2948-2955.

[26] C. Soto, S. Bi, and A. K. Roy-Chowdhury, "Distributed multi-target tracking in a self-configuring camera network," in Proc. IEEE Conf. CVPR, Jun. 2009, pp. 1486-1493.

[27] IEEE, "IEEE 802.15.4-2006 IEEE Standard for Information technologyTelecommunications and information exchange between systems - Local and metropolitan area networks - Specific requirements Part 15.4: Wireless Medium Access Control (MAC) and Physical Layer (PHY) Specifications for Low Rate Wireless Personal Area Networks (LRWPANs)," IEEE Computer Society, pp. 1-160, 2005. 
[28] S. C. Ergen. (2004, Sep. 10). ZigBee/IEEE 802.15.4 Summary [Online]. Available: http://www.eecs.berkeley.edu/csinem/academic/publications/ zigbee.pdf

[29] S. Boyd and L. Vandenberghe, Convex Optimization. Cambridge, U.K.: Cambridge Univ. Press, 2004

[30] X. Jiang, J. Polastre, and D. E. Culler, "Perpetual environmentally powered sensor networks," in Proc. 4th Int. Symp. IPSN, Apr. 2005, pp. 463-468.

[31] R. Madan, S. Cui, S. Lall, and A. Goldsmith, "Cross-layer design for lifetime maximization in interference-limited wireless sensor networks," IEEE Trans. Wireless Commun., vol. 5, no. 11, pp. 3142-3152, Nov. 2006.

[32] S.-J. Kim, X. Wang, and M. Madihian, "Distributed joint routing and medium access control for lifetime maximization of wireless sensor networks," IEEE Trans. Wireless Commun., vol. 6, no. 7, pp. 2669-2677, Jul. 2007.

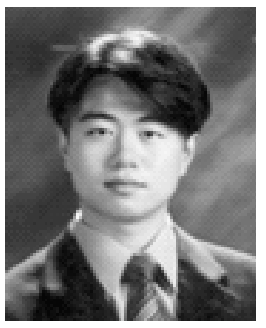

Sungjin Lee was born in Korea, in 1980. He received the B.S. degree from Soongsil University in 2005 and the M.S. and Ph.D. degrees from Yonsei University in 2007 and 2011, respectively, all in electrical engineering. Currently, he is with Samsung Electronics on $5 \mathrm{G}$ communication development. His research interests are in the area of wireless communication networks, especially in sensor network, small-cell network, and wireless resource management issues.

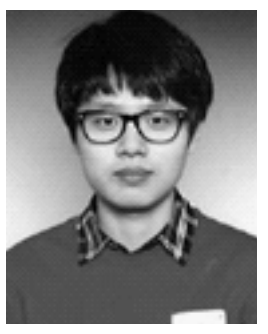

Inwoong Lee was born in Korea, in 1990. He received the B.S. degree in electrical engineering from Yonsei University in 2012, where he is currently pursuing the M.S. and Ph.D. degrees at the Multidimensional Insight Laboratory, Yonsei University. His research interests are in the areas of multiuser MIMO systems, interference management, and wireless multimedia communications.

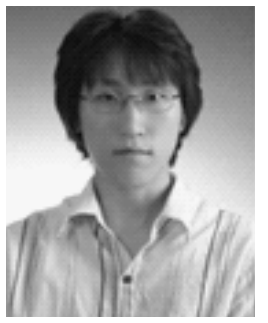

Seonghyun Kim was born in Korea, in 1985 . He received the B.S. degree in electrical engineering from Yonsei University in 2009, where he is currently pursuing the M.S. and Ph.D. degrees with the Multidimensional Insight Laboratory. His research interests are in the areas of multiuser MIMO systems, interference management, and cooperative communications.

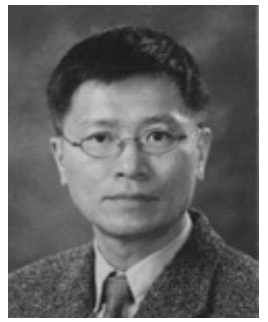

Sanghoon Lee (M'05-SM'12) received the B.S. degree in electrical engineering from Yonsei University in 1989 and the M.S. degree in electrical engineering from the Korea Advanced Institute of Science and Technology in 1991. From 1991 to 1996, he was with Korea Telecom. He received the $\mathrm{Ph} . \mathrm{D}$. degree in electrical engineering from the University of Texas at Austin in 2000. From 1999 to 2002, he was with Lucent Technologies on 3G wireless and multimedia networks. In 2003, he joined the Faculty Member of the Department of Electrical and Electronics Engineering, Yonsei University, Seoul, Korea, where he is a Full Professor. He has been an Associate Editor of the IEEE TRANSACTIONS ON IMAGE PROCESSING since 2010, an Editor of the Journal of Communications and Networks since 2009, and the Chair of the IEEE P3333.1 Quality Assessment Working Group since 2011. He currently serves as the Technical Committee of the IEEE IVMSP, the General Chair of the 2013 IEEE IVMSP Workshop, and a Guest Editor of the IEEE TRANSACTIONS ON IMAGE PROCESSING in 2013. He has received the 2012 Special Service Award from the IEEE Broadcast Technology Society and the 2013 Special Service Award from the IEEE Signal Processing Society. His research interests include image/video quality assessments, medical image processing, cloud computing, wireless multimedia communications, and wireless networks.

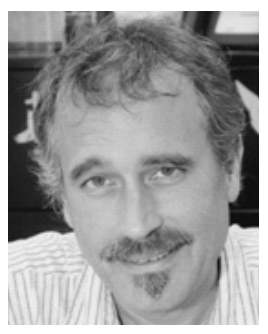

Alan Conrad Bovik is the Curry/Cullen Trust Endowed Chair Professor with the University of Texas at Austin, where he is the Director of the Laboratory for Image and Video Engineering. He is a Faculty Member with the Department of Electrical and Computer Engineering and the Center for Perceptual Systems, Institute for Neuroscience. His research interests include image and video processing, computational vision, and visual perception. He has published more than 650 technical articles and holds two U.S. patents. His several books include the recent companion volumes The Essential Guides to Image and Video Processing (Academic Press, 2009).

He has received a number of major awards from the IEEE Signal Processing Society, including: the Best Paper Award in 2009, the Education Award in 2007, the Technical Achievement Award in 2005, and the Meritorious Service Award in 1998. He was a recipient of the Honorary Member Award of the Society for Imaging Science and Technology for 2013, received the SPIE Technology Achievement Award for 2012, and was the IS\&T/SPIE Imaging Scientist of the Year for 2011. He received the Hocott Award for Distinguished Engineering Research at the University of Texas at Austin, the Distinguished Alumni Award from the University of Illinois at Champaign-Urbana in 2008, the IEEE Third Millennium Medal in 2000, and the two Journal Paper Awards from the International Pattern Recognition Society in 1988 and 1993. He is a fellow of the Optical Society of America, the Society of Photo-Optical and Instrumentation Engineers, and the American Institute of Medical and Biomedical Engineering. He has been involved in numerous professional society activities, including: Board of Governors, the IEEE Signal Processing Society from 1996 to 1998, a Co-Founder and Editor-in-Chief of the IEEE TRANSACTIONS ON IMAGE PROCESSING from 1996 to 2002, an Editorial Board of The PRoceEdings of THE IEEE from 1998 to 2004, a Series Editor for Image, Video, and Multimedia Processing (Morgan and Claypool) since 2003, and a Founding General Chairman of the First IEEE International Conference on Image Processing, Austin, TX, in 1994.

Dr. Bovik is a registered Professional Engineer in the State of Texas and is a frequent consultant to legal, industrial, and academic institutions. 